\title{
Pair Creation in the Pulsar Magnetosphere
}

\author{
Paul N. Arendt, Jr. \\ parendt@aoc.nrao.edu \\ Jean A. Eilek \\ jeilek@aoc.nrao.edu \\ Department of Physics, New Mexico Tech, Socorro, NM 87801
}

\begin{abstract}
We present numerical simulations of the electron-positron plasma creation process in a simple neutron star magnetosphere. We have developed a set of cascade 'kernels', which represent the endpoint of the pair cascades resulting from monoenergetic photon seeds. We explore two popular models by convolving these kernels with the seed photon distributions produced by curvature radiation and by inverse Compton scattering. We find that the pair plasma in either case is well-described in its rest frame by a relativistic Maxwellian distribution with temperature near $m c^{2} / k_{B}$. We present cascade multiplicities and efficiencies for a range of seed particle energies and stellar magnetic fields. We find that the efficiencies and multiplicities of pair creation are often lower than has been assumed in previous work.
\end{abstract}

\section{INTRODUCTION}

An electron-positron pair plasma is a key ingredient in most models of pulsar radio emission. This plasma is assumed to come from a pair cascade which occurs in the open field line region close to the star's magnetic axis. In this region, rotation-induced electric fields pull charged particles from the polar cap and accelerate them to relativistic energies $\left(\gamma \lesssim 10^{7}\right)$. These particles radiate 'seed' $\gamma$-ray photons, either by curvature emission (Sturrock 1971; Ruderman \& Sutherland 1975; Arons 1983), or by inverse Compton scattering of ambient thermal photons (Bussard, Alexander, \& Mészáros 1986; Daugherty \& Harding 1986; Sturner \& Dermer 1994). In the strong magnetic fields of pulsars, these primary photons are susceptible to magnetic one-photon electron-positron pair creation (Tsai \& Erber 1974). The newly formed leptons in turn radiate 'secondary' photons, most commonly through synchrotron radiation (Harding \& Preece 1987). The secondary photons may be capable of further pair production. As Sturrock (1971) first pointed out, this cycle of energetic photon emission and further pair creation continues, forming a 'pair production avalanche', which ends only when all remaining photons are transparent to pair creation.

Whether this pair plasma forms or not, and its properties when it does, are crucial issues in models of the radio emission region. The plasma properties determine the possible wavemodes which the plasma can support. Excitations of these wavemodes ultimately become the radio emission we observe (after coupling to electromagnetic modes and escaping the magnetosphere). Propagation of these modes through the pair plasma may leave its signature on the observed signal (through dispersive effects). In addition, the plasma itself may be the source of the excitation of the waves which lead to radio emission (if there is free energy available in the plasma distribution at birth to drive instabilities). 
The need for a quantitative understanding of the pair plasma distribution function (DF) has been apparent in the literature for some time. Existing calculations of plasma wavemodes and propagation have either assumed analytically convenient DFs, without physical justification, or have tried to quantify a plausible heuristic DF introduced by Arons (1981). (Examples include Buschauer \& Benford 1975, Arons \& Barnard 1986, Beskin, Gurevich, \& Istomin 1988; Kazbegi, Machabeli, \& Melikidze 1991; Weatherall 1994, Lyubarskii 1996; Gedalin, Melrose, \& Gruman 1998; Lyutikov, Blandford, \& Machabeli 1999). It is therefore critical to determine the properties of the plasma created by a pair-production avalanche in the pulsar magnetosphere. This is the primary focus of our paper.

In addition to leptons, the pair cascade can also produce high-energy photons. A small number of pulsars exhibit such high-frequency emission. Some authors (e.g., Romani 1996; Horotani \& Shibata 1999) believe that this emission comes from a high-altitude 'outer gap' active region, others (e.g., Rudak \& Dyks 1999; Zhang \& Harding 2000) believe that this emission originates in the polar cap region. We therefore include photon spectra as a secondary focus in the cascade models we present in this paper.

\subsection{The Setting: the Polar Flux Tube}

To place our calculation into a larger context, we briefly summarize the standard model of the pulsar magnetosphere. (We follow, for instance, Ruderman \& Sutherland 1975, Arons 1992, or Melrose 1992, 1995).

Soon after pulsars were discovered, Goldreich \& Julian (1969) pointed out that a pulsar magnetosphere would not be empty. A rotating magnetized neutron star in vacuum generates electric fields strong enough to overcome the star's binding energy for electrons (and possibly light ions), contradicting the vacuum assumption. Most of the inner magnetosphere is now assumed to corotate with a 'force-free' electric field $\mathbf{E}_{f f}=-c^{-1}(\boldsymbol{\Omega} \times \mathbf{r}) \times \mathbf{B}$, filled with the corotational (Goldreich-Julian) charge density $\rho_{G J}=(4 \pi)^{-1} \nabla \cdot \mathbf{E}_{f f}$, where $\boldsymbol{\Omega}$ is the angular velocity of the star. The exception is on those field lines which extend beyond the 'light cylinder' (where the corotational speed is equal to $c$ ), defining the 'polar flux tube' (Arons 1983). Radio emission is thought to originate in the plasma within this polar flux tube.

The polar flux tube's active properties are due to the extension of its $\mathbf{B}$ field lines beyond the light cylinder. Charged particles which stream outward along these lines are unable to maintain a corotational force-free state along the entire field line (even within the light cylinder). (In the 'closed' magnetosphere, where B lines do not cross the light cylinder, B lines become electric equipotentials.) Although individual particles in the flux tube may escape to form a stellar wind, polar currents are assumed to cross field lines somewhere near the light cylinder and complete a circuit back to the star to preserve its overall neutrality.

The details of how these polar currents return to the star are unknown. This is unfortunate, because the global current structure is crucial for determining the accelerating electric fields, and thus the photon seeds for the pair production cascade (if it occurs). If a cascade occurs, the pair plasma may allow the premature 'shorting out' of the accelerating potential (e.g. Arons \& Scharlemann 1979; Shibata, Miyazaki, \& Takahara 1998). This in turn modifies the conditions assumed to seed the cascade in the first place.

\subsection{Modeling The Pair Cascade}

The pair cascade takes place within this setting. We have already noted the complexity of a fully selfconsistent solution. Lacking this, important factors for the cascade development are uncertain. What is the 
energy of the primary beam charges? By what means do these primary charges produce seed $\gamma$-ray photons? In contrast, the microphysics underlying the cascade is well-known. We know precisely the differential QED cross sections for lepton and photon production: the local $\mathbf{B}$ field, photon energy and impact angle determine the outcome.

Daugherty and Harding (1982; 'DH82' hereafter) modeled the cascade numerically. Their simulations began with a single particle streaming out along the $\mathbf{B}$ field, whose curvature radiation began the cascade. They studied the $\gamma$-ray photon distribution produced by the cascade, with passing reference to the properties of the underlying plasma.

Our focus is different. Because we are especially concerned with the properties of the pair plasma, we wanted to extend previous work to determine those properties, and their dependence on magnetospheric parameters (magnetic field and primary beam energy). Thus, we set up our calculation to determine both the momentum distribution function (DF) and the density of the pair plasma relative to that of the primary beam.

Because we were concerned about the uncertainties implicit in global magnetosphere models, we took a new approach to the cascade. We wrote a code which follows, in time and space, the cascade induced by a monoenergetic population of photons. We include pair production by the photons and synchrotron radiation by the leptons. Each run terminates when all particle and photon production ceases. At the end of each run, we store the photon and lepton distributions (typically about $10^{5}$ particles per run, binned into 50-100 momentum bins, depending on the number of particles available). We treat each such run as a 'kernel'. The final cascade is then formed as the composite of many such runs, each weighted by the relative distribution of seed photons of that particular energy. In this paper we form composite cascades assuming the seed photons come from curvature radiation, or from inverse Compton scattering of ambient X-ray photons.

In the remainder of this paper, we describe our code ( $\S 2$; with some details in the Appendix); give a qualitative overview of the pair cascade process $(\S 2)$, and present the results of our parameter-space survey $(\S 3)$. Our primary results are presented in $\S 4$, where we form composite cascades, based on photon seeds from curvature radiation and inverse Compton scattering. We close in $\S 5$ with a summary and some final comments. The reader interested only in final plasma or photon distributions might skip the details and jump to $\S \S 4$ and 5 .

\section{OUR KERNELS: MONOENERGETIC SEEDS}

\subsection{Structure of the Calculation}

Our simulations began with photons injected over a polar cap, at the stellar surface (actual seed photon formation at a finite but low altitude will encounter a similar physical environment). The magnetic field geometry is assumed to be dipolar. The photons are monoenergetic (to within one percent) with energy $\varepsilon$. The initial locations are uniformly scattered in area on the stellar surface over the entire polar cap (a circle centered on the magnetic pole, of radius $r_{*} \sqrt{\Omega r_{*} / c}$, where $r_{*}$ is the stellar radius). The photons are distributed uniformly in a cone of half-angle $\theta_{\max }$ relative to the local $\mathbf{B}$ direction.

The major input parameters for each run are: the magnetic field strength, $B_{*}$ (which we describe in terms of the field value at the magnetic pole, at the altitude corresponding to the photon seed emission), the energy $\varepsilon$ of the primary photons seeding the cascade, and their maximum initial angle $\theta_{\max }$ (to which we refer using $\left.\mu=1-\cos \theta_{\max }\right)$. The range of these parameters covered by our complete set of monoenergetically-seeded 
cascades was determined partly by the demands of the specific model cascade seed mechanisms of $\S 4$, and partly by our performing a systematic exploration over a wide range of these parameters (to determine the influence of each parameter on the cascade process). Our complete set of simulations covered initial photon energies $\varepsilon \equiv \hbar \omega / m c^{2}$ (where $m$ is the electron mass) from 50 to $10^{7}$, initial field values $B_{*}=10^{11}, 10^{12}$, and $10^{13} \mathrm{G}$, and cosine complements $\mu$ from 0 up to 0.1 . A subset of these results is presented in this section, with parameters chosen from a representative sample of the range covered. The smallest opening angles shown here correspond to $\mu=10^{-12}$; we found that lowering $\mu$ beyond this usually had no further effect on the cascade (the exceptions to this were the $B_{*}=10^{13}$ runs at the largest initial photon energies $\varepsilon$ that we investigated; there, smaller $\mu$ lowered the cascade multiplicities somewhat). Angles larger than the Lorentz beaming cone, $\theta_{\max } \sim 1 / \gamma$ (where $\gamma$ is the Lorentz factor of the beam particles emitting the initial photons), are not believed to be relevant in present-day models of the pulsar pair cascade (which all rely upon a high-energy particle beam to emit or scatter the seed photons). We nevertheless included some larger angles in our monoenergetic runs to investigate the variation of cascade possibilities (but did not use them in our composite models, presented in $\S 4$, which were all from a simulated particle beam). We present them here in case future models include primary photons at high angles (such as might be expected from energetic return currents impacting the star when inverse Compton braking is negligible).

Our code follows the position and momentum of each photon as it propagates away from the star. To determine when a photon pair creates, we also track the optical depth, $\tau$, for each photon. For photons, $\tau$ is the fraction of a mean free path traversed before pair production occurs, so that $\tau=1$ represents a $1 / e$ probability that it has not disappeared and created a pair. We split the simulation into discrete timesteps (with duration chosen so that the optical depth of no photon increases by more than $\delta \tau=0.2$ per timestep). The photons thus begin by propagating outward unattenuated, until some of them exceed $\tau=1$ and create electron-positron pairs.

The local conditions for each photon (field strength, particle energy and angle) determine how much its $\tau$ is incremented at each timestep. For photons of energy $\varepsilon$, the main relevant parameter is $\chi$, defined as (Daugherty \& Harding 1983)

$$
\chi=\frac{\varepsilon}{2} B^{\prime} \sin \theta
$$

where $\theta$ is the angle between the photon's direction and $\mathbf{B}$, and $B^{\prime}=B / B_{c r}$ is the dimensionless magnetic field strength, The 'critical' magnetic field strength is $B_{c r} \equiv m^{2} c^{3} / e \hbar=4.4 \times 10^{13} \mathrm{G}$. The total attenuation coefficient $R(\chi)$ (given fully in the Appendix), can be approximated at low $\chi$ values by

$$
R(\chi) \approx 0.23 \frac{\alpha}{\lambda_{C}} B^{\prime} \exp \left(-\frac{4}{3 \chi}\right),
$$

where $\alpha$ is the fine-structure constant, and $\lambda_{C}$ is the Compton wavelength of the electron.

When we exceed $\tau=1$ for a photon, that photon is destroyed and an electron-positron pair created in its place. The properties of the new leptons are determined probabilistically, using numerical approximations to the differential attenuation coefficients for pair production into the possible lepton states. Expressions for these coefficients are taken from Daugherty \& Harding (1983), and included in the Appendix.

The possible states of the created pair depend upon the state of the parent photon, allowed states of charged leptons in strong magnetic fields, and relevant conservation laws. The energy levels $E /\left(m c^{2}\right)$ of an electron or positron with momentum $\mathbf{p} /(m c)$ in a uniform $B$ field are given by (Johnson \& Lippman 1949)

$$
E_{n, s, p_{\|}}^{2}=1+p_{\|}^{2}+B^{\prime}(2 n \pm s+1)
$$


where $p_{\|}=\mathbf{p} \cdot \mathbf{B} / B$ is momentum along $\mathbf{B}, s$ can be \pm 1 and specifies the spin state, and $n$ is a nonnegative integer specifying the orbital quantum number (Landau level). The leptons may be born into an excited Landau level and emit high-energy synchrotron radiation, which can create further lepton pairs. After the rapid synchrotron emission a newly created lepton emits in these strong fields, it will be in the ground state $(n=0$ and $1 \pm s=0)$, constrained to move along $\mathbf{B}$.

We also track the position and momentum of each lepton, constraining its guiding center to move along B. To decide when to create a new synchrotron photon, we use a semi-classical method to simulate the lifetime of the parent lepton's current quantum state, and use this to define a mean free path. Details are given in the Appendix. As with the photons, we use the fraction of a local mean free path traversed during each timestep to increment $\tau$. When $\tau>1$, we create a single synchrotron photon. Keeping track of emission times is most important in weaker $\mathbf{B}$ fields $\left(B \ll B_{c r}\right)$, as all synchrotron emission is extremely rapid in strong fields.

For leptons in the strong magnetic fields of pulsars, the quantized nature of synchrotron emission cannot be ignored. The energy difference between adjacent Landau levels is $m c^{2} B^{\prime}$, so synchrotron photons can easily exceed $\varepsilon=2$ in strong fields, and in weaker fields when the leptons are born with high $\mathbf{p}_{\perp}$ (where $\mathbf{p}_{\perp}$ is the momentum at right angles to $\mathbf{B}$, implictly defined by the Landau level of eq. [3]). As discussed in Harding \& Preece (1987), the classical synchrotron spectrum can give erroneous results under these strong B conditions: the high-frequency limit of the classical spectrum can violate conservation of energy, and the low-frequency classical spectrum can extend below the minimum allowed transition. As the leptons may lose $\mathbf{p}_{\perp}$ either by emission of many low-energy photons or a few high-energy ones, we choose the photon energy probabilistically. This is done using the differential synchrotron spectrum in Harding \& Preece (1987). The Appendix gives some details of this spectrum (eq. [A3]) and the code implementation of these processes.

A simulation has reached completion when all photons satisfying $\varepsilon>2$ have essentially zero probability of pair creation, and all leptons have radiated away all $\mathbf{p}_{\perp}$ by synchrotron emission.

Some parameters affect the cascade only very weakly for a given initial photon injection; for uniformity, we left these fixed. In all the runs presented here, we set the neutron star radius at $10 \mathrm{~km}$, with a rotation period of $33 \mathrm{~ms}$. The angle between the rotation and magnetic dipole axes was set at $45^{\circ}$. Of these fixed parameters, the cascade details will be most sensitive to the rotation period, due to the more strongly curved field lines available above the larger polar caps of faster pulsars.

Note that we have ignored several effects which may have a small effect on the cascade. For simplicity, we ignored polarization and spin dependence in particle creation processes, and only used cross-sections which have been averaged over polarization and spin. We ignored general relativistic effects, such as frame dragging and gravitational refraction. Frame dragging leads to higher beam energies (through making accelerating electric fields stronger), but beam energy is just a parameter in our (composite) simulations anyway. We did not include any electric fields in these simulations. The primary effect a nonzero $\mathbf{E}_{\|}$would have in our simulations is to cause relative streaming of the leptons of different charge. Another effect is that pair production efficiency is boosted in the presence of an $\mathbf{E}$ field (Daugherty \& Lerche 1975). Including this effect is locally equivalent to increasing $B$, a variation we do investigate here. We ignored the possibility of bound positronium formation in strong $\left(B>B_{c r}\right.$ ) fields (e.g. Usov \& Melrose 1996), the decay of which may be more likely to result in photon multiplication than creation of a (stable) free pair. We also ignored photon splitting (a closely related process, differing only in that the intermediate pair is virtual). Qualitatively, the inclusion of these higher-order processes might be expected to lower the pair creation efficiencies in strong fields, and degrade the final photon spectrum to lower energies. 


\subsection{A Typical Monoenergetic Cascade}

The simulation results will be easier to understand if we first sketch the qualitative features of some typical monoenergetic runs. We choose three simulations: all have initial photon energy $\varepsilon=10000$ and cosine complement $\mu=10^{-6}$. Although this is a larger angle than is allowed by $1 / \gamma_{b}$ beamed emission from a 'primary' beam as in most models, we can illustrate a wider variety of possible cascade behavior than with runs at only very small angles. (We again emphasize that we respected $1 / \gamma_{b}$ beaming in our composite runs of $§ 4$.) These illustrative runs differ from each other only in the surface magnetic field strength $B_{*}$ at the magnetic pole: $10^{11}, 10^{12}$, and $10^{13} \mathrm{G}$, respectively.

\subsubsection{Particle production}

In figures 1 and 2 we illustrate the factors governing the existence and development of a typical cascade. In each figure, pair creation events are shown in the left panels, and synchrotron photon creation events in the right panels. In addition to the spatial development of the cascade, these two figures illustrate the two factors which govern pair creation: opacity and photon energy.

In figure 1 we show the spatial development of the cascade, plotting magnetic polar angle against (log) altitude for each pair creation or energetic $(\epsilon>2)$ synchrotron photon creation event. (Throughout this work, we use $\varepsilon$ to denote seed photon energies, and $\epsilon$ for general photon energies, including post-cascade.) Figure 2 is similar to figure 1, but instead of magnetic polar angle we show $\log \epsilon_{\perp}$ as the ordinate, where $\epsilon_{\perp}$ refers to the perpendicular frame energy of the parent photon for pair creation events, and that of the daughter photon for synchrotron emission events. Several features of these figures are worth pointing out.

Figure 1 shows that the cascade in a $10^{13} \mathrm{G}$ field has two steps: a low-altitude burst of pair formation and synchrotron emission, followed by a high-altitude burst of pair creation without further photon creation. In the two lower-field cases, the cascade has only one burst, in which both pair and photon creation occur.

Inspection of figure 2 clarifies the two factors which control the onset of the cascade. Consider the available energy $\left(\epsilon_{\perp}\right)$ of the photon which pair creates. In all creation events in the lower field runs, and in the lower-altitude creation events in the $10^{13} \mathrm{G}$ run, $\epsilon_{\perp} \gg 2$. These photons have more than enough energy to create a pair. The leptons created in these events are born in high Landau levels (they have finite angles relative to $\mathbf{B}$ ). They will therefore create new photons through synchrotron radiation; the short lifetime of this process is reflected in the spatial bunching of photon creation events, close to the location of the original pair event. In contrast, the high-altitude second burst in the $B=10^{13} \mathrm{G}$ field comes from photons with $\epsilon_{\perp} \simeq 2$. These creation events come from photons traveling nearly tangent to $\mathbf{B}$. Their propagation angles, relative to $\mathbf{B}$, increase at higher altitudes due to field line curvature, until they finally attain $\epsilon_{\perp} \geq 2$, at which point pair creation occurs. The created leptons are born traveling tangent to $\mathbf{B}$, in the ground Landau state, so that they cannot create any further synchrotron photons. Thus the high-altitude pair creation burst is not accompanied by further photon creation.

We can understand this behavior quantitatively. The high-field, high-altitude creation events are governed by energetics. They illustrate the first condition necessary for pair creation, that the photon must have at least the rest mass energy measured in the perpendicular frame:

$$
\epsilon_{\perp}=\epsilon \sin \theta \geq 2
$$

By contrast, the lower-field, lower-altitude creation events are coverned by opacity. They can be under- 

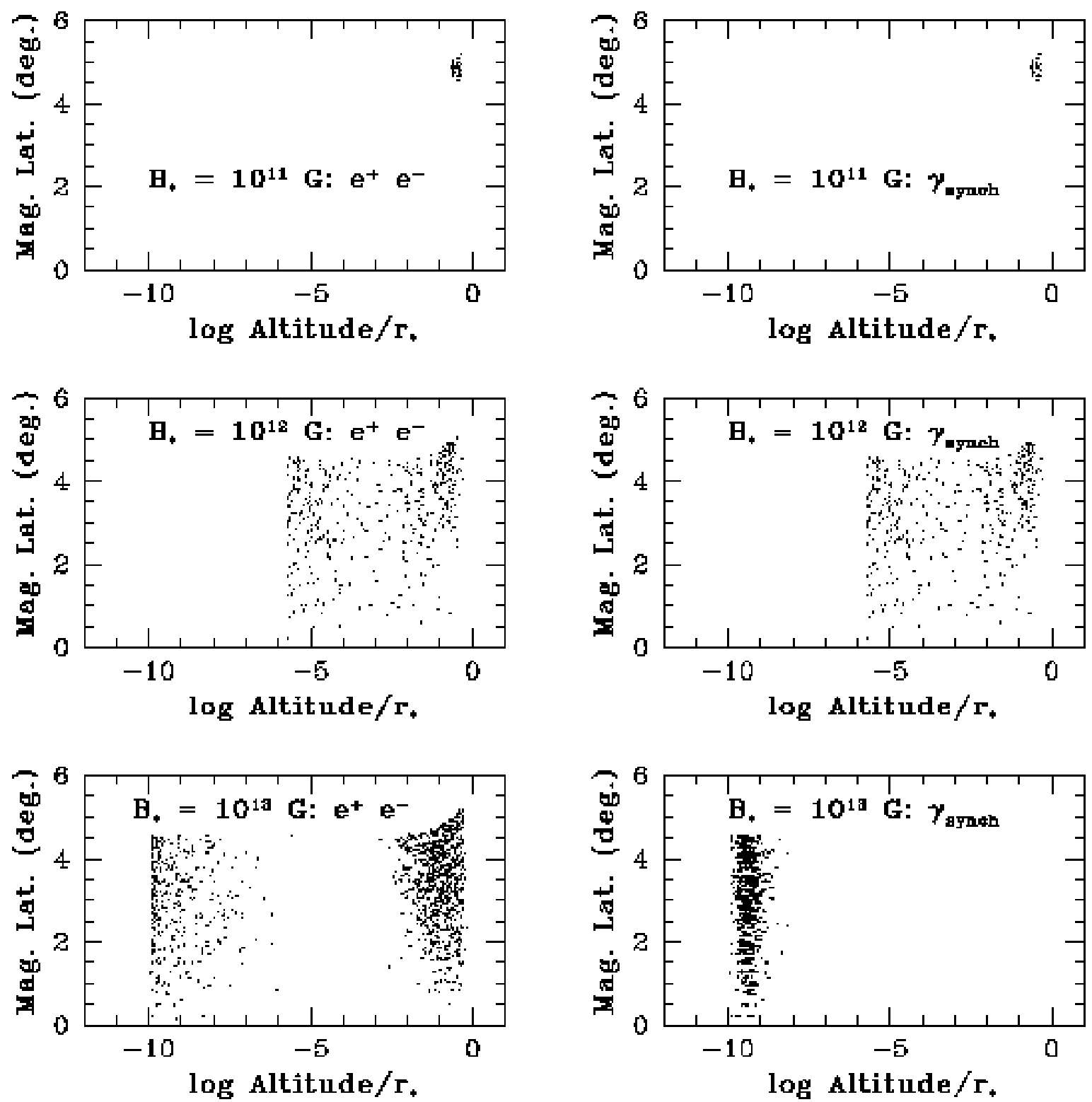

Fig. 1.- Locations of Particle Production. Magnetic latitude $\rho$ is plotted against log altitude (in stellar radii), for photons of $\varepsilon=10000$ injected into a cone with angle parameter $\left(\mu=1-\cos \theta_{\max }=10^{-6}\right)$. The left column shows sites of lepton pair creation, and the right column shows sites of energetic $(\epsilon>2)$ synchrotron photon emission. The number of starting photons was 300,200 , and 300 for $B_{*}=10^{11}, 10^{12}$, and $10^{13} \mathrm{G}$, respectively. At smaller angles (e.g., for $1 / \gamma_{b}$ beaming), the only difference in this diagram is the disappearance of the low-altitude burst of pairs and synchrotron photons in the $10^{13} \mathrm{G}$ field. 

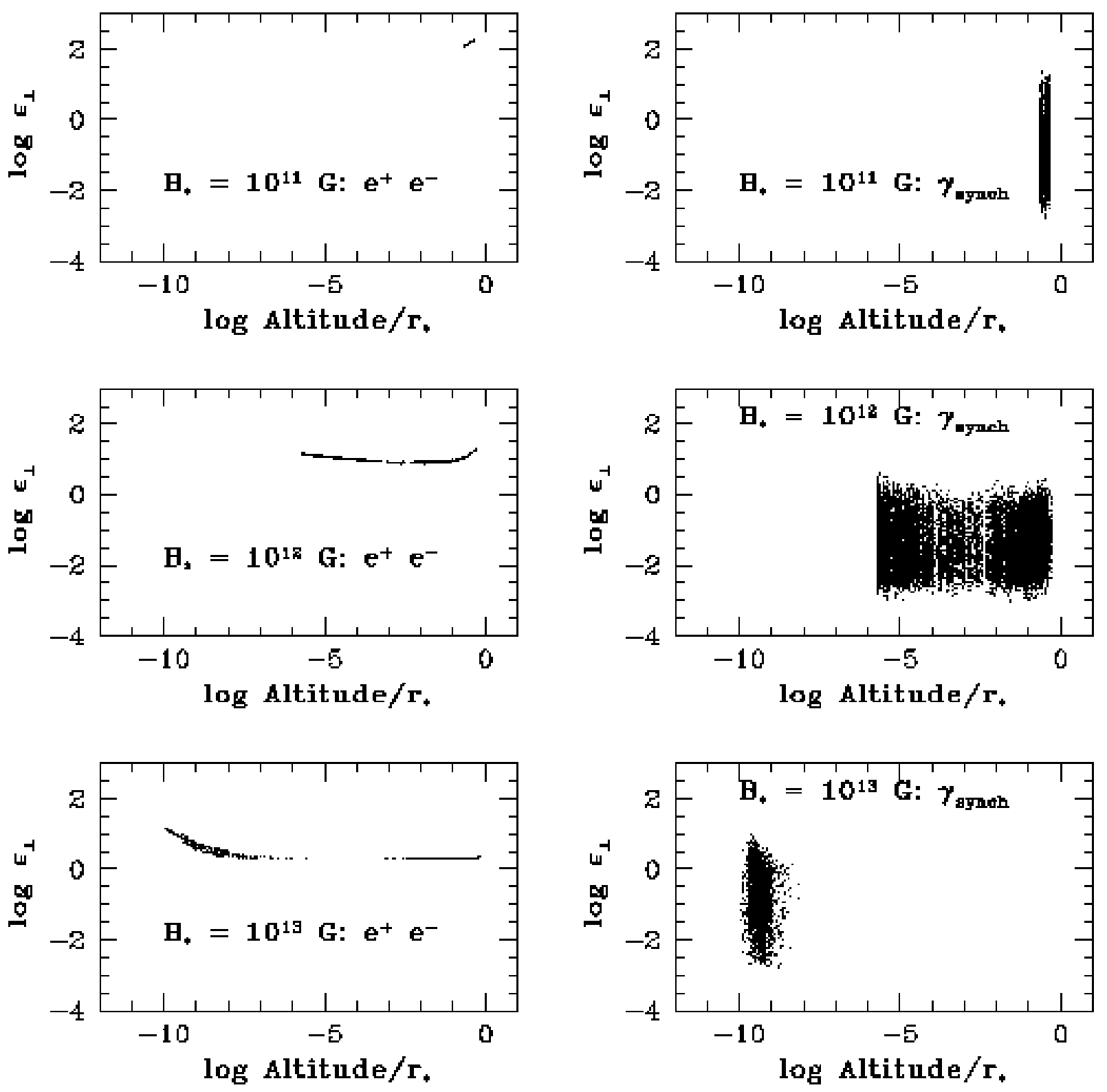

Fig. 2.- Photon Energies in Particle Production. Similar to figure 1, but showing photon $\epsilon_{\perp}$ (energy in a frame where $\mathbf{k} \cdot \mathbf{B}=0$ ) versus altitude. The energy shown is that of the parent photon for the pair production events, and of the emitted photon for the synchrotron events. Note that many synchrotron photons are often emitted right at the sites of pair creation seen in figure 1. It is evident that many of these secondary photons are born with insufficient $\epsilon_{\perp}(<2)$ to create another pair, unless their angle with $\mathbf{B}$ increases significantly. 
stood from the low- $\chi$ behavior of $R(\chi)$, given in equation (2). Based on our numerical results, and noting that $B$ decreases as $\left(r / r_{*}\right)^{3}$, we can generously estimate that a stellar radius will be the maximum distance traversed before pair creation. Setting the mean free path (the inverse of $R$ ) equal to $10 \mathrm{~km}$ gives a minimum value of $\chi$ realistically necessary for pair production:

$$
\chi_{m} \approx\left(23.5+0.75 \ln B^{\prime}\right)^{-1} \approx 0.05 .
$$

(This depends only weakly on $B^{\prime}$, so it can be assumed robust when changing the magnitude or geometry of the field). Opacity to pair creation thus requires $\chi>\chi_{m}$. Using equation (1), we find the second condition necessary for pair creation:

$$
\epsilon_{\perp} B \gtrsim 0.1 B_{c r} \sim 4 \times 10^{12} G .
$$

Our runs at $B_{*}=10^{11}$ and $10^{12} \mathrm{G}$ are thus opacity limited: the photons pair create when they propagate to angles so that $\epsilon_{\perp} \gtrsim 0.1 B_{c r} / B$. This relation describes the locus of pair creation sites in the two lower $B_{*}$ diagrams of figure 2 , and the low-altitude creation sites in the $B=10^{13} \mathrm{G}$ run. In contrast, the high-altitude pair creation events in our $B_{*}=10^{13} \mathrm{G}$ example are energy limited. The photons nearly always satisfy $\chi>\chi_{m}$, but they cannot pair create until they also satisfy $\epsilon_{\perp}>2$.

In addition to the cascade onset, the cascade termination is worth comment. In all cases (including those not shown), the cascade finished before the particles reached one stellar radius above the surface (or injection point). Thus, pair cascade is a rapid process, with the time from seed photon emission to cascade completion being less than $r_{*} / c \sim 30 \mu$ sec. If seed photon production is a sporadic process, then the pair plasma created should be expected to also exist only sporadically at any given point in the polar flux tube. The short completion time is reminiscent of the temporal, 'microstructure' fluctuations seen in pulsar signals (e.g. Hankins 1996). We also note that changing the geometry of the $\mathbf{B}$ field would have to be significant over these short length scales, in order to have any noticable effect on the cascade. Finally, figure 1 also shows an enhanced probability of pair production towards the edge of the polar flux tube. This is of course due to the higher field line curvature there, and is reminiscent of 'conal' emission (as defined in Rankin 1983).

\subsubsection{The cascade at other angles}

Most of the features of the examples presented apply to the cascade at smaller seed photon opening angles. Figures 1 and 2 lose only the low-altitude creation events in the $10^{13} \mathrm{G}$ field (which includes all synchrotron photons in that case). The cascade remains essentially unchanged for all leptons whose first pair creation burst was opacity-limited at smaller angles, including down to $\mu=0$.

At larger $\mu$, most of the initial pair creation events are opacity-limited. Copious synchrotron photons are created in the first pair creation burst in all fields, leading in turn to many further pair creation events in further generations. We shall see in the next section that this does not necessarily imply a greater efficiency of energy transfer from the primary beam to the pair plasma, since the excess synchrotron photons which do not pair create wind up with a large portion of the energy budget.

\section{GENERAL RESULTS: MONOENERGETIC KERNELS}

In this section we present a representative subset of the general results of our monoenergetic simulations. The set of runs shown span cosine complements $\mu$ from 0.1 down to $10^{-12}$, stellar magnetic fields $B_{*}$ of 
$10^{11}, 10^{12}$, and $10^{13} \mathrm{G}$, and initial photon energies $\varepsilon$ from 300 to $10^{5}$.

\subsection{Efficiency of Plasma and Photon Creation}

The efficiency of the cascade is important to global models of the pulsar magnetosphere as well as to models of the radio and high-energy photon emission. Our monoenergetic runs allow us to extract both number and energy conversion efficiencies. Since an arbitrary number of starting photons seed each kernel run, these efficiencies are defined per seed photon.

We begin with the pair plasma. To describe its density, we use the conversion ratio diagnostic $C^{l}\left(\varepsilon ; \mu, B_{*}\right)$, defined as the number of pairs produced per parent photon. This can be roughly thought of as the number of generations of leptons produced in the cascade. This quantity will also serve as a weighting function for determining plasma parameters of composite cascades, which begin with a spectrum of initial photon energies.

Table 1 shows $C^{l}$, the lepton creation efficiencies found in our runs. We see that $C^{l}$ increases with $B_{*}$ and $\varepsilon$. This is as expected. For low $\varepsilon, \mu$, and $B_{*}$ the seed photons' mean free path for pair creation is larger than the scale height of the magnetosphere, so they escape intact and then $C^{l}=0$. For a fixed $B_{*}$, we find that $C^{l}$ is primarily a function of the (maximum) pair creation parameter of the initial photons. This initial parameter, $\chi_{i}$, is defined using equation $(1)$ as $\chi_{i}=\chi\left(\varepsilon, B_{*}, \theta_{\max }(\mu)\right)$, where $\theta_{\max }=\arccos (1-\mu)$ is the largest angle of the injected photons. $C^{l}$ increases with angle for large angles, but at small angles it often saturates at some value $C^{l} \lesssim 1$. This occurs when the primary photons are unable to pair produce until they propagate far enough outward to increase their angle with $\mathbf{B}$. This $C^{l} \lesssim 1$ regime - for low field values and small angles - is roughly limited by $\chi_{i} \lesssim 0.01$. (We shall see later that the lepton DFs for these low- $C^{l}$ runs are independent of the opening cosine complement $\mu$ ). For larger $\chi_{i}$ values, we find that a dual power law $C^{l} \propto \theta_{\max }^{a} \varepsilon^{b}$ fits the $C^{l}$ data nicely, with different values of $a$ and $b$ for different values of $B_{*}$. We find $(a, b) \approx(0.5,0.8)$ for $B_{*}=10^{13}$ and $(a, b) \approx(0.4,0.5)$ for the lower $B_{*}$ runs.

We can form another diagnostic by dividing the final plasma energy by the initial photon energy, to define an efficiency of energy transfer, $E^{l}\left(\varepsilon ; \mu, B_{*}\right)$, from primary photons into the leptons. Table 1 also lists these values. It is important to note that, contrary to the assumptions made in some current models, $E^{l}<1$ is commonly the case. The missing energy corresponds to photons which escape the magnetosphere. The parameters which determine the energy efficiency are $B_{*}$ and the cosine complement $\mu$. $B_{*}$ determines the overall range of efficiencies: $E^{l} \sim 0-.03, \sim 0-.35$, and $\sim .40-1.0$ for $B_{*}=10^{11}, 10^{12}$, and $10^{13} \mathrm{G}$, respectively. Synchrotron losses thus weigh heavily in the polar cap's energy budget if the magnetic field is weak. In this limit, the efficiency increases with decreasing opening angle at first, but drops off again when the angle becomes small enough for many of the seed photons to escape without producing pairs. Remarkably, the efficiency is highly insensitive to the initial photon energy $\varepsilon$ (except at the pair creation threshold where $C^{l} \leq 1$, where lowering $\varepsilon$ causes more seed photons to escape).

We also find that $E^{l}$ is roughly anticorrelated with $C^{l}$ for a fixed $B_{*}$, and peaks at small $\mu$ and large $\varepsilon$. That is, many pairs are created only when relatively little of the available energy goes into making pairs. This is understandable since copious synchrotron radiation accounts for both effects, but unfortunate if one's polar cap model requires an efficient creation of dense plasma. Interestingly, the lepton energy efficiency is relatively insensitive to the initial photon energy. Fixing other parameters, lowering $B_{*}$ always lowers $E^{l}$, and lowers $C^{l}$ except at very large $\varepsilon$ where $C^{l}$ peaks for $B_{*}=10^{12} \mathrm{G}$. 
The photons which escape are important both as a diagnostic and as a direct observable. To quantify the photon count, we calculate the photon conversion ratio, $C^{p}\left(\varepsilon ; \mu, B_{*}\right)$, defined as the number of escaping photons with $\epsilon \geq 2$ divided by the initial number of seed photons. Table 2 lists the values of $C^{p}$ of the simulations, which ranged from zero to several hundred. Several trends are evident in the table. Not surprisingly, $C^{p}$ always decreases with decreasing angle, since the first generation of leptons produced must have large $\mathbf{p}_{\perp}$ at birth for many synchrotron photons to be produced. For a fixed angle, $C^{p}$ usually increases with increasing $\varepsilon$. The few exceptions to this were all in the $10^{13} \mathrm{G}$ field, where some escaping primary photons were more likely to create pairs as $\varepsilon$ was increased, lowering $C^{p}$. Finally, we note that $C^{p}$ was a maximum for the intermediate value of $B_{*}=10^{12}$, holding the other parameters fixed. This behavior is attributable to the fact that we need both efficient pair creation (which requires a large $B_{*}$ ) and copious synchrotron emission by the created leptons (which happens for low $B_{*}$ ) to produce many escaping synchrotron photons.

The efficiency of energy transfer into energetic photons, $E^{p}$, is also presented in Table 2. $E^{p}$ is defined as the ratio of the energy in escaping photons with $\epsilon>2$ to the seed photon energy. The difference between unity and the sum of $E^{p}$ and $E^{l}$ represents the fractional energy that went into photons softer than $\epsilon=2$. In the weakest field $\left(10^{11} \mathrm{G}\right)$, most of the primary photon energy remains in hard photons, even at large $\varepsilon$ and large $\mu$. Also in the weak field, $E^{p}$ increases with decreasing opening angle, since photons are less likely to pair create at small angles. In the strongest field $\left(10^{13} \mathrm{G}\right)$, almost none of the primary photon energy remains in hard photons. Decreasing $\mu$ tends to decrease $E^{l}$ in strong fields, since synchrotron emission disappears for small angles there. In our intermediate field $\left(10^{12} \mathrm{G}\right)$, low-energy seed photons mimic the weak-field trends for $E^{l}$, and high-energy seed photons mimic the strong-field trends.

The cascade at $10^{11} \mathrm{G}$ (in a dipole field) is in fact so dominated by photons that following just those with the potential to pair create rapidly ate up available computing resources. For that reason, the statistics we obtained on leptons in the $10^{11} \mathrm{G}$ field are relatively poor compared with those in the stronger fields, and we did not include the $10^{11} \mathrm{G}$ field in our composite simulations of $\S 4$.

\subsection{Plasma Distribution Functions}

From a radio emission perspective, the shape of the final lepton DFs is one of the most interesting results of the cascade simulation. The shape of the DF determines the nature and propagation of wavemodes in the plasma and possible instabilities which may be converted into radio emission.

Although we generated too many DFs in our parameter-space survey to present them all here, we can describe the general behavior concisely. The DF shape is overwhelmingly controlled by the opening cosine complement $\mu$, with secondary dependence on magnetic field and seed photon energy. The shape of the DF tends to saturate at both large and small angles.

We present a typical cross-section of the DFs for the entire range of $\mu$ for fixed $\varepsilon$ and two different $B_{*}$ values in figure 3. The saturated large-angle DF mentioned above is demonstrated by the $\mu=0.1 \mathrm{DFs}$ in the figure. This $\mathrm{DF}$ is flat from $10^{-4} \leq p_{\|} \leq 1$, and drops off roughly as $\exp \left(-p_{\|}{ }^{0.2}\right)$ above this. This limiting large-angle shape is independent of magnetic field and seed photon energy. As we decrease $\mu$, the DFs become narrower and peak at higher momentum. At very small angles (where $C^{l}$ is 1 ), these DFs again saturate to a single shape, but now this shape depends on both $B_{*}$ and $\varepsilon$. The double-humped shape of some of the low-angle DFs in figure 3 is due to photons which give the members of a created pair opposite $p_{\|}$ in the $\mathbf{k} \cdot \mathbf{B}=0$ frame to conserve $p_{\|}$; the difference is amplified by the boost to the star's reference frame. 


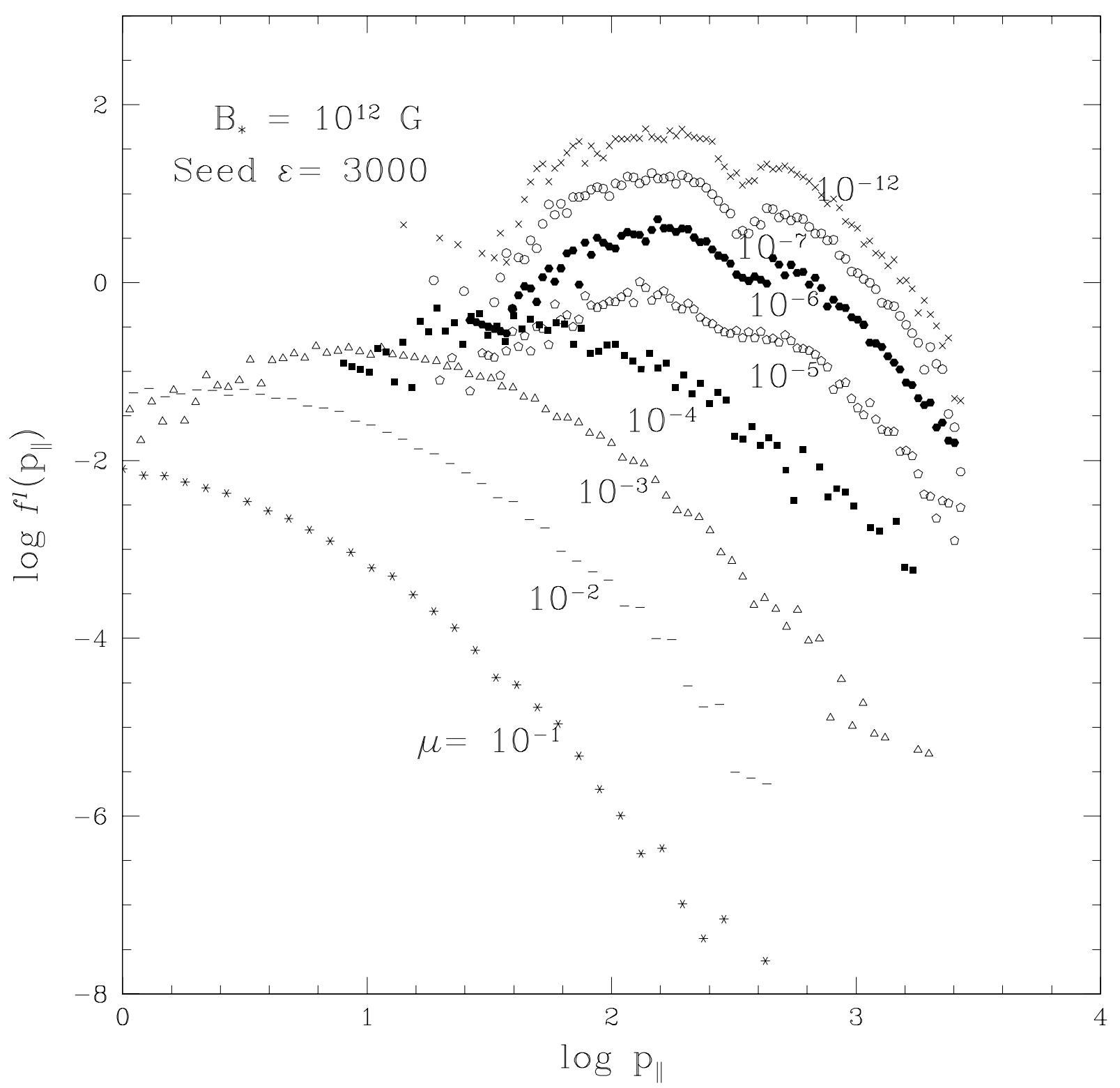

Fig. 3.- (a) Lepton DF Dependence on Angle Parameter $\mu$ : $B_{*}=10^{12}$ G. Vertical offsets have been made for clarity. Notice how the low- $\mu$ DFs all agree; the simulation results for low $\mu$ are in fact identical to the case $\mu=0$. 


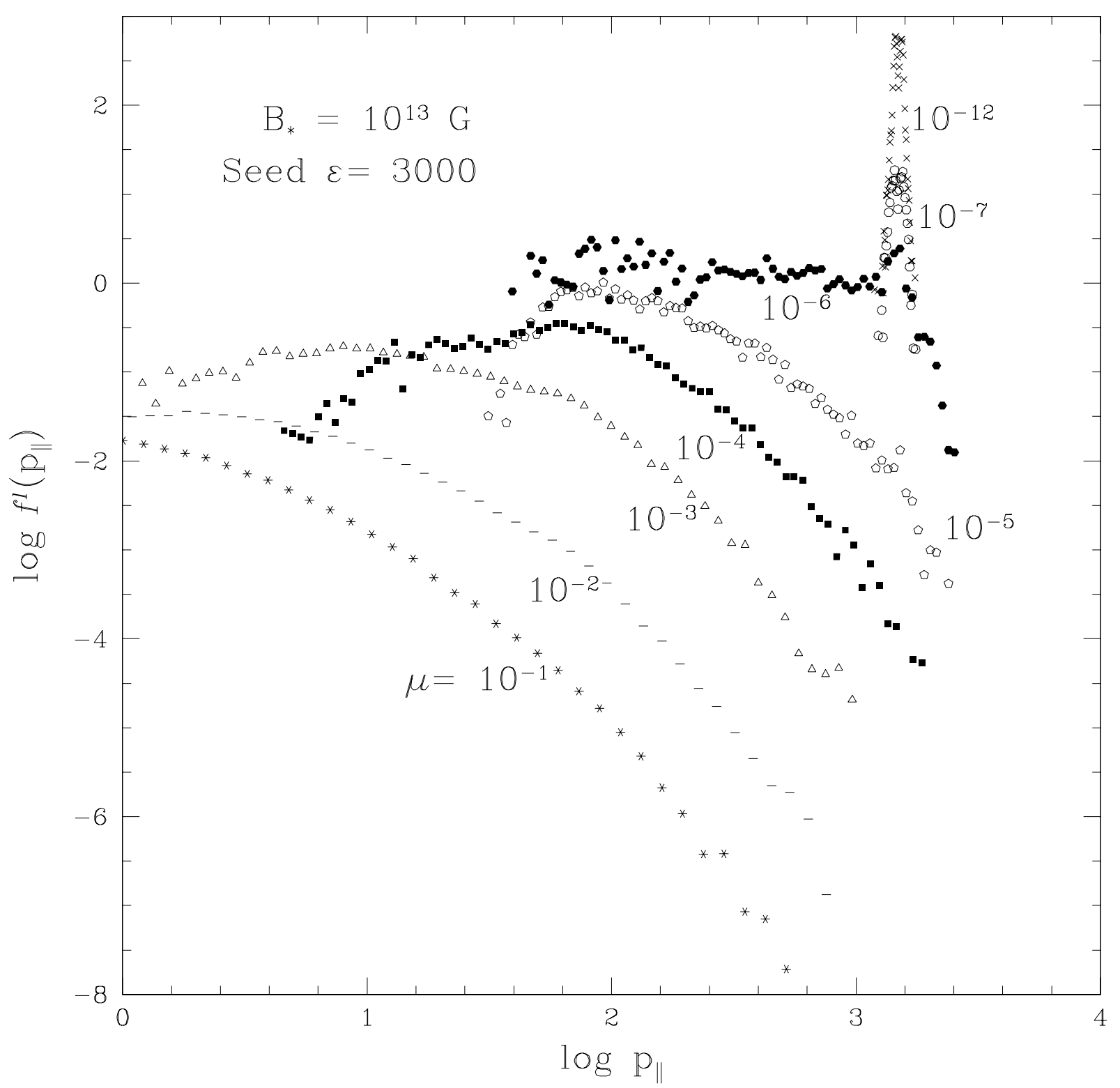

Fig. 3.- (b) Same as (a), for $B_{*}=10^{13}$ G. Note that the low- $\mu$ DFs are much narrower than those in (a). In contrast to (a), there is a slight $\mu$ dependence to the width of the low- $\mu$ DFs: narrower angles produce narrower DFs. 
As low angles are the relevant ones for seed photons in most models, we illustrate the dependence of low-angle DFs on $B_{*}$ and on $\varepsilon$ in figure 4 . The important point illustrated here is that the width and other features in the shape are determined by $B_{*}$, while the location of the peak is determined by $\varepsilon$.

Since we wished to use these lepton DFs from monoenergetic seed photons to generate more realistic DFs (by convolution), we determined an empirical expression which fits the monoenergetic DF shapes. We fit only the DFs for the $B_{*}=10^{12}$ and $10^{13} \mathrm{G}$ runs, due to the poor plasma creation efficiencies in the $10^{11}$ $\mathrm{G}$ runs. We found that a parabola in $\log f\left(p_{\|}\right)-\log p_{\|}$space is a remarkably good fit to most of the DFs. We therefore used the expression

$$
f\left(p_{\|}\right)=N \exp \left[-K\left(\ln \frac{p_{\|}}{p_{0}}\right)^{2}\right]
$$

where $K$ and $p_{0}$ are fit parameters to be determined for each 'kernel' run. This describes the fractional number of particles $f\left(p_{\|}\right)$having final momenta (along $\mathbf{B}$ ) between $p_{\|}$and $p_{\|}+d p_{\|}$. Thus, $f\left(p_{\|}\right)$combines leptons from all generations of the cascade. The normalization constant $N=\sqrt{K / \pi} e^{4 K} / p_{0}$ is chosen to ensure that $\int_{0}^{\infty} f\left(p_{\|}\right) d p_{\|}=1$ (this will be used in the composite simulations presented in $\S 4$ ).

We carried out least-squares fitting to determine the optimal values of the parameters $p_{0}$ (location of the peak of $f$ ) and $K$ (roughly, the inverse of the 'width' of $f$ ). The fits were empirically very good over most of the parameter space, excepting the very largest opening angles (which are not used in the composite runs).

As examples, the least-squared values for $p_{0}$ and $K$ for $\mu=10^{-6}$ and $\mu=10^{-12}$ are plotted in figure 5. The lines connecting the fits represent interpolations used when simulating the composite DFs of $\S 4$. Values of $p_{0}$ ranged from below 1 to almost 200 for $B_{*}=10^{12}$, and were very nearly half the parent photon momentum for $B_{*}=10^{13}$. Values of $K$ went from 0.1 to 0.8 for the lower $B_{*}$, and were much larger (10 to several hundred) for the higher $B_{*}$. Note that the values of $K$ peaked at photon energies of a couple thousand, then dropped at larger energies. At large $\varepsilon$, the photons can produce pairs with a larger spread of characteristics, which widens the DF. Over the range where $K$ is dropping, equation (7) was a poorer fit than elsewhere. Also, at very large energies $\left(\varepsilon>10^{5}\right)$ in the $10^{13} \mathrm{G}$ field, some pairs were born in excited Landau levels, and synchrotron radiation then created further pairs (as in the weaker-field cases). However, these latter generations of leptons were spread over a wide range of low $p_{\|}$in the DF, and equation (7) no longer described the DF shape well. We did not consider seed photon energies above $\left(\varepsilon=10^{5}\right)$ in the $10^{13}$ $\mathrm{G}$ field in $\S 4$ for this reason.

\subsection{Photon Spectra}

Distribution functions can be also be created for the escaping photons. To avoid confusion with the lepton DFs, we refer to these as spectra throughout this work. However, it is prudent to emphasize that these represent the result of binning together photon number counts, not energies.

The photon spectra which come from our simulations have fewer trends worth mentioning than the plasma DFs; to first order, all are roughly the same shape. The photon spectra are all either power laws which steepen at some $\epsilon_{\text {break }}$, or everywhere convex functions which may or may not exhibit steepening at some $\epsilon_{\text {break }}$. The power laws have slopes of -.5 to -2 below the break, and steepen to as much as -4 above it. The convex spectra have local slopes in the same ranges as the power laws. The steeper power laws generally appear for the low field and large opening angles and shallower power laws for the high field and small opening angles. For the $B_{*}=10^{12}$ runs, none of the photon spectra exhibited a true power law over 

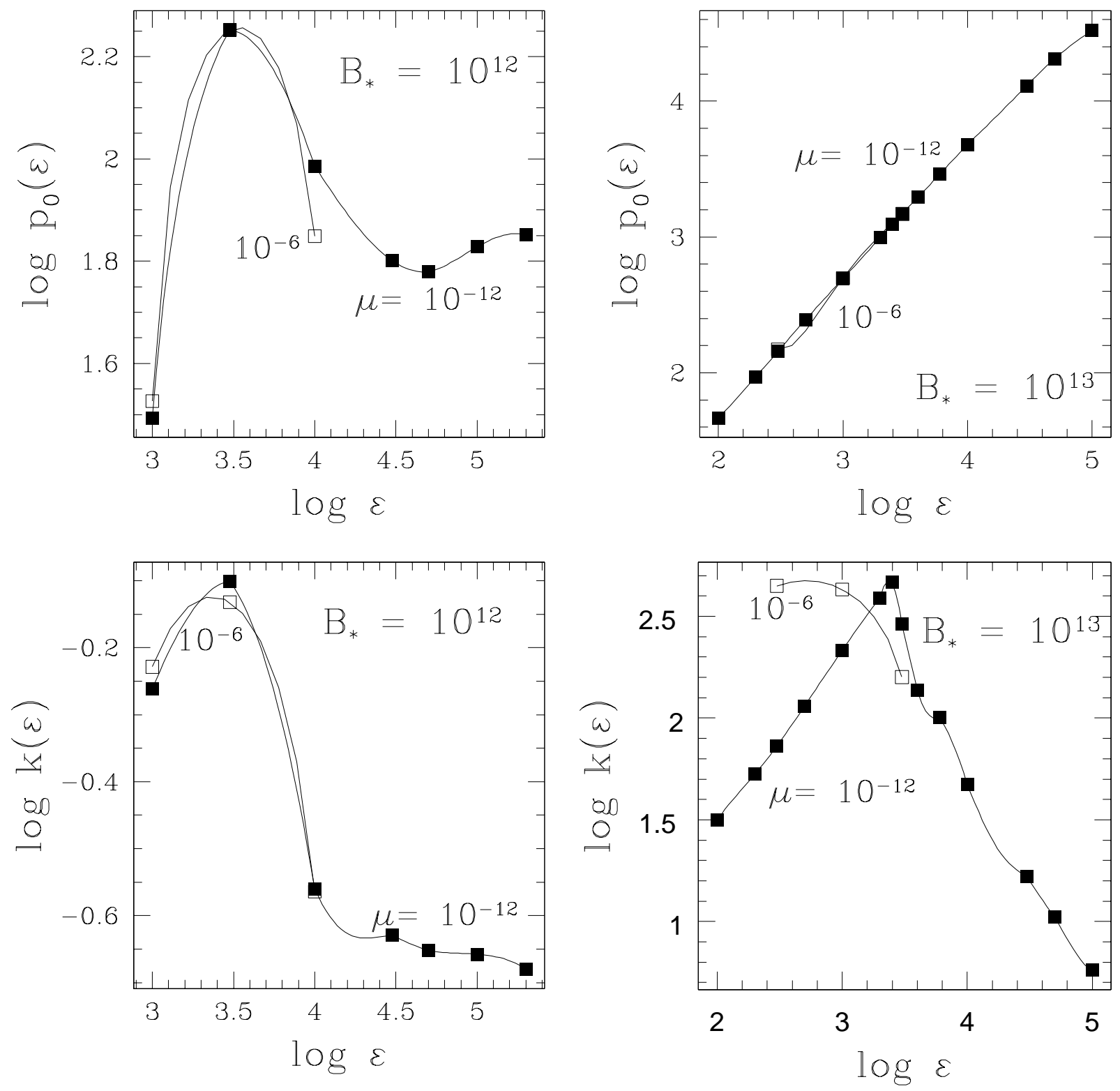

Fig. 4.- Lepton DFs for $\mu=10^{-7}$. The $B_{*}=10^{12}$ and $10^{13} \mathrm{G}$ DFs are shown for cosine complement $\mu=10^{-7}$ and two values of seed photon energy. A characteristic double-humped shape is evident, and is due to unequal splitting of parent photon energy to the lepton pair. The much more narrow DFs in the $10^{13}$ $\mathrm{G}$ case give the much larger $K$ values seen in figure 5 . Vertical offsets have been made for clarity. 


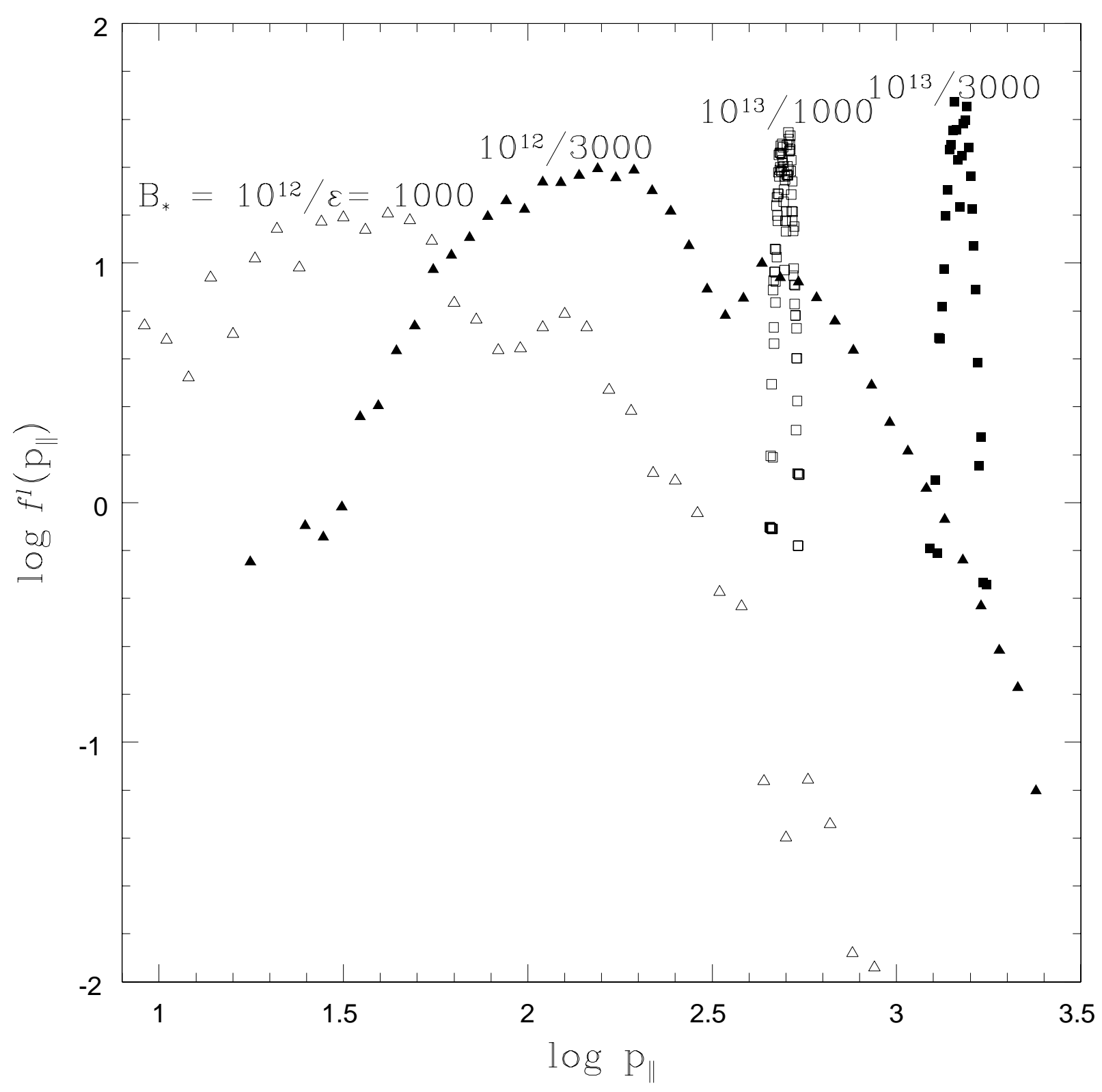

Fig. 5.- Lepton DF Fit Parameters. Lepton DFs (viewed in the star's frame) were least-square fit (in log space) to a function of the form $f\left(p_{\|}\right) \propto \exp \left[-K\left(\ln p_{\|} / p_{0}\right)^{2}\right]$. The best-fit values of these two parameters are shown: $p_{0}$ is roughly the mean parallel momentum of the DF, while $K$ is an inverse width. Actual fits are shown as points, while the lines show interpolations used for the composite DFs of $\S 4$. Two values of $\mu$ are shown; the larger angle was only considered for lower-energy seed photons. 
any significant range.

Samples of the convex photon spectra for several values of $\mu$ for a fixed $B_{*}$ and $\varepsilon$ are shown in figure 6 . Except for small changes in slope, the spectra all look remarkably similar. Below the break, if it occurs, the spectra are steeper for larger $\mu$. This is consistent with the results of Beskin et al. (1993), who found that photon spectra steepened with each succeeding generation of parent leptons (our largest angles produce the largest $C^{l}$, which is roughly the number of lepton generations). Above the break, the spectra are steeper for smaller $\mu$, curving the spectra even more. The bump at $\epsilon=3000$ is just the primary photons which escaped the magnetosphere without producing a lepton pair.

We can empirically (and somewhat arbitrarily) classify the photon spectra according to type (power law or convex). The power laws only seem to occur for the large field and small angles (with a steep spectrum), and for the low field and large angles (with a shallower spectrum). A sample of the different spectral types is given in figure 7, which illustrates a sample power law with break and two convex spectra with breaks. It is evident that the distinction between a power law and convex spectrum is somewhat fuzzy, as for the $B_{*}=10^{12} \mathrm{G}$ case. Note that these spectra differ from each other only by the parameter $B_{*}$. In large fields, the break on the photon spectrum is due to pair creation by the highest-energy synchrotron photons. In low fields, the break is due to the intrinsic synchrotron spectrum steepening. The break locations ranged from about $\epsilon \simeq 40-10^{3}$ for the lower two values of $B_{*}$, increasing with increasing energy and generally with decreasing angle. For $B_{*}=10^{13}$, the break location was near $\epsilon=100$ over most of the parameter space, but decreased slightly for large angles and low seed photon energies. At smaller $\mu$, the synchrotron photons disappear in the $B_{*}=10^{13} G$ case, and thus so does the relevant spectrum of figure 7 .

\section{APPLICATIONS TO PULSAR CASCADE MODELS}

Our monoenergetic runs gave us several useful quantities for cascades deriving from monoenergetic photons. We found the distribution functions, $f^{l}\left(p_{\|}\right)$and $f^{p}(\epsilon)$ (for leptons and photons, respectively). We also found conversion ratios $C^{l}(\varepsilon)$ and $C^{p}(\varepsilon)$, and energy efficiencies $E^{l}(\varepsilon)$ and $E^{p}(\varepsilon)$. Cascades in pulsars are hardly likely to derive from monoenergetic photons, however. Many authors have assumed that the photons which initiate the cascade are generated by curvature radiation. An interesting, more recent, alternative uses inverse Compton scattering to seed the cascade. In this section we present the results of using our monoenergetic results as 'kernels' to determine the cascades resulting from these two mechanisms.

We do this by convolving our results with the energy distribution of seed photons which start the cascade, which we call $N(\varepsilon)$. We follow the standard picture in assuming that charges are pulled from the star and accelerated to Lorentz factor $\gamma_{b}$ (these are the 'beam' charges). We do not attempt to determine $\gamma_{b}$ self-consistently, but rather treat it as a parameter. To create seed photons, we consider both curvature radiation and magnetic resonant inverse Compton scattering by the beam charges.

For both of these processes, the characteristic length over which a charge radiates most of its energy is not too different from the scale of the polar cap and pair formation region (Sturner 1995). We therefore take $N(\varepsilon)$ to be the composite photon spectrum (for the appropriate process) emitted by a beam charge as it radiates most or all of its energy. The specific definitions of $N(\varepsilon)$ are given below in $\S 4.1$ and $\S 4.2$.

Once we know $N(\varepsilon)$, we can find the composite photon and lepton distributions from

$$
F^{p}(\epsilon)=\int f^{p}(\epsilon ; \varepsilon) N(\varepsilon) C^{p}(\varepsilon) d \varepsilon
$$




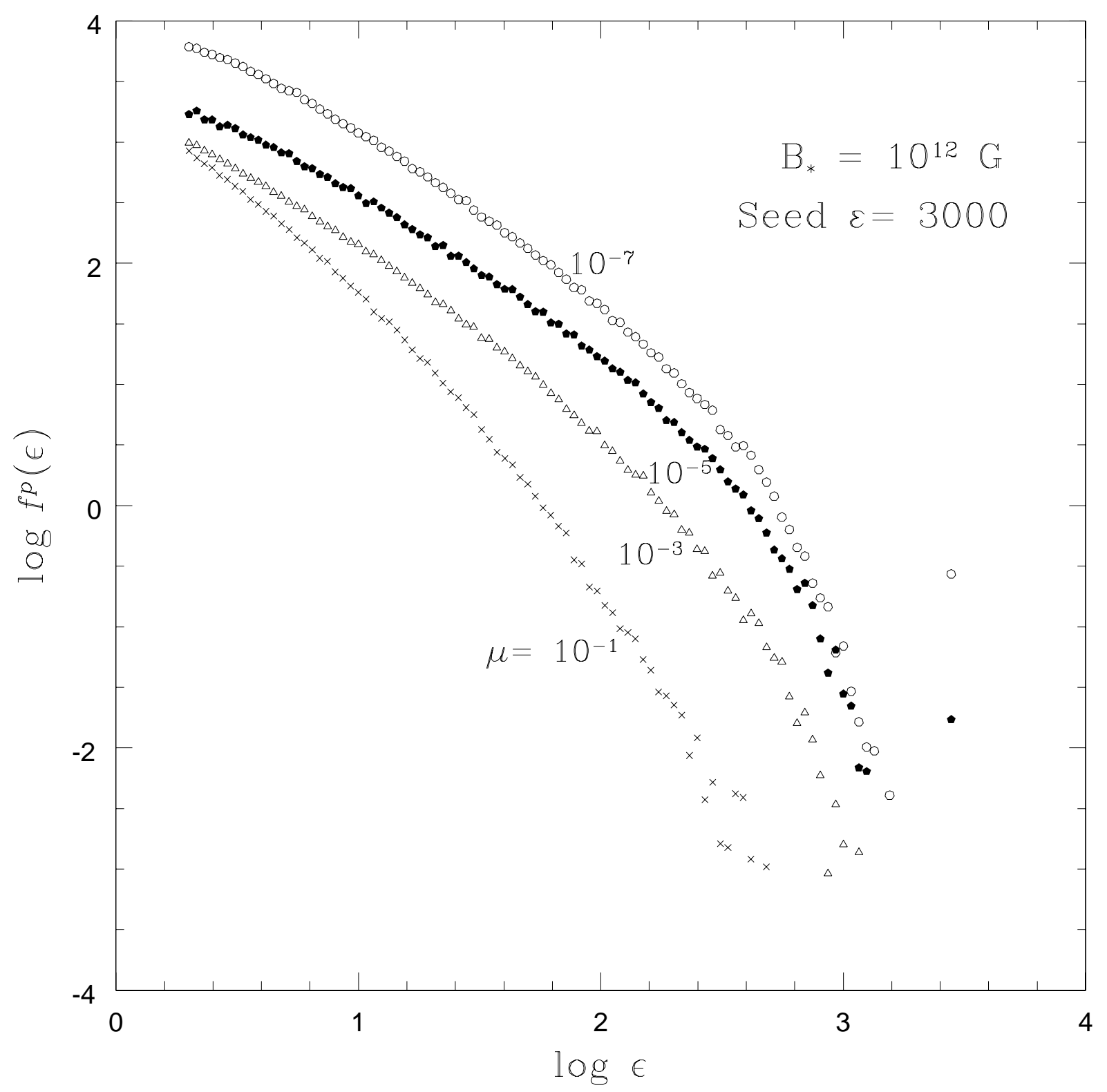

Fig. 6.- Photon Spectrum Dependence on $\mu$. A sample of photon spectra is shown for $B_{*}=10^{12} \mathrm{G}$ and seed photon energy $\varepsilon=3000$, for various cosine complements $\mu$. Spectra for angles smaller than $\mu=10^{-7}$ are identical to the spectrum shown for $\mu=10^{-7}$. Vertical offsets have been made for clarity. These typify the spectral shapes found: a gradual steepening with energy is seen, often with a high-energy break above which the spectrum sharply steepens. The points at $\epsilon=3000$ are seed photons which did not create pairs. 


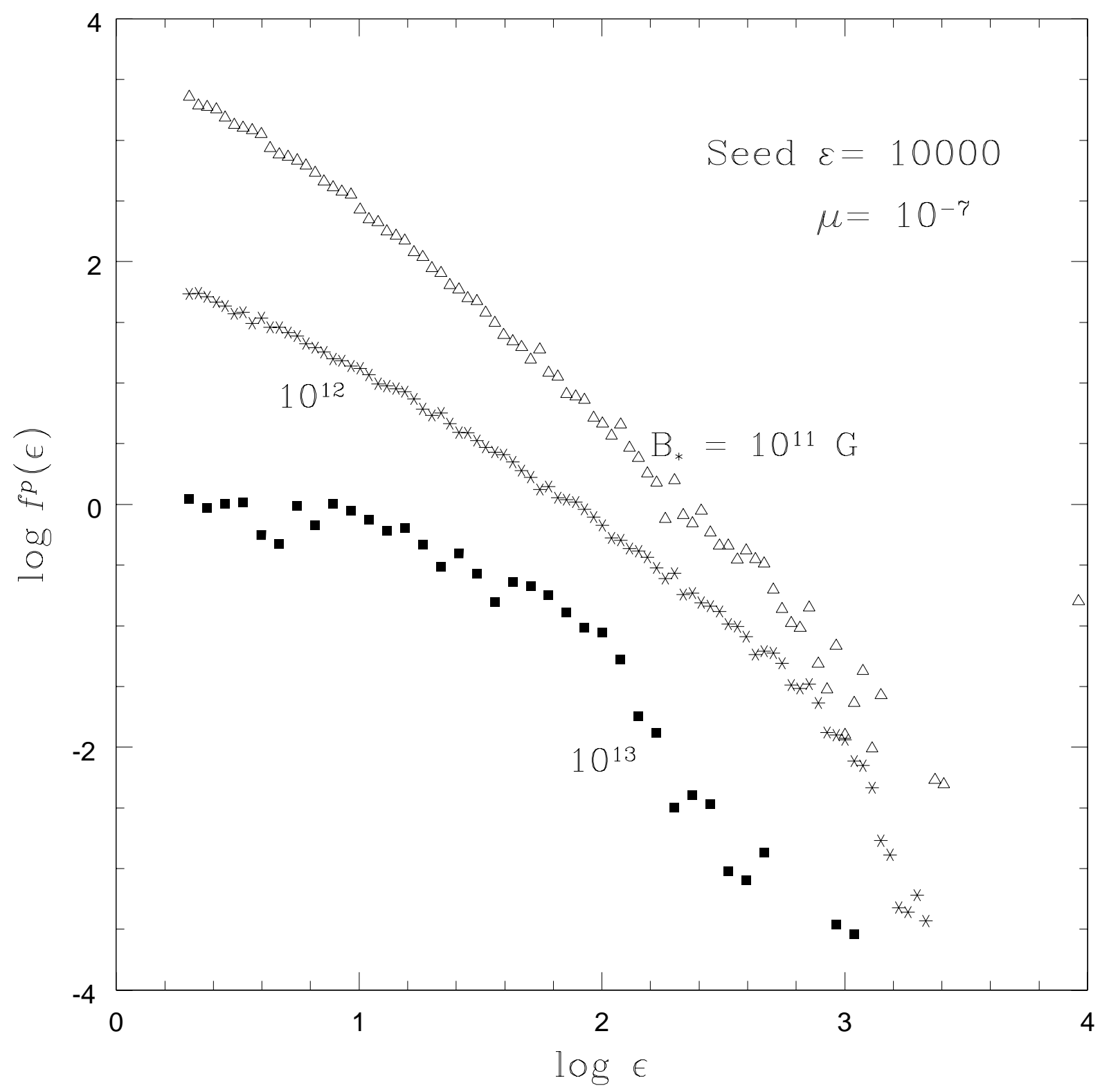

Fig. 7.- Photon Spectrum Dependence on $B_{*}$. Seed photon energy $\varepsilon$ was $10^{4}$, and opening cosine complement $\mu$ was $10^{-7}$. The high-energy breaks evident where the high- $B_{*}$ DFs steepen are due to pair creation losses. Statistics are poor for the $B_{*}=10^{13} \mathrm{G} \mathrm{DF}$; at even smaller $\mu$ there are no secondary photons in the strong field. The DFs have been offset vertically. 
and

$$
F^{l}\left(p_{\|}\right)=\int f^{l}\left(p_{\|} ; \varepsilon\right) N(\varepsilon) C^{l}(\varepsilon) d \varepsilon .
$$

(Recall that $\varepsilon$ refers to seed photon energies, while $\epsilon$ is used for post-cascade photon energies.) To evaluate the lepton DFs, we had to deal with the strongly varying shapes of the monoenergetic DFs $\left(f^{l}\left(p_{\|}\right)\right)$. To do this, we used our analytic approximations to the simulated DFs, described in $\S 3.2$, and evaluated the integral in equation (9) numerically. Parameters used in the analytic approximations were interpolated for values of $\varepsilon$ between those actually simulated (shown by the lines in fig. [5]). As the monoenergetic photon spectra were simpler, we dealt with them more simply. We binned the seed photons from a particular $N(\varepsilon)$ around the $\varepsilon$ values for which we performed monoenergetic runs, and used these results to evaluate the integral in equation (8) by discrete summation.

We computed number counts and energy efficiencies for the composite runs in a similar fashion. We define the multiplicity as the number of pairs (half the number of leptons) created per primary beam particle:

$$
M^{l}=\int F^{l}\left(p_{\|}\right) d p_{\|}=\int N(\varepsilon) C^{l}(\varepsilon) d \varepsilon .
$$

For photons, the multiplicity is the number of energetic photons created per primary beam particle:

$$
M^{p}=\int_{\epsilon>2} F^{p}(\epsilon) d \epsilon=\int N(\varepsilon) C^{p}(\varepsilon) d \varepsilon .
$$

We define fractional power as the ratio of energy escaping (in leptons or in photons) to $\gamma_{b}$, the energy of a primary beam particle:

$$
P^{l}=\frac{1}{\gamma_{b}} \int F^{l}\left(p_{\|}\right) \gamma\left(p_{\|}\right) d p_{\|}=\int N(\varepsilon) E^{l}(\varepsilon) d \varepsilon
$$

and

$$
P^{p}=\frac{1}{\gamma_{b}} \int F^{p}(\epsilon) \epsilon d \epsilon=\int N(\varepsilon) E^{p}(\varepsilon) d \varepsilon
$$

In the composite runs we took $\theta_{\max } \approx 1 / \gamma_{b}$, or $\mu \sim 1 /\left(2 \gamma_{b}^{2}\right)$, for a particular value of primary beam energy $\gamma_{b}$. We remind the reader that, although not stated explicitly in the above expressions, all calculated quantities depend upon the surface field strength, $B_{*}$.

\subsection{Curvature Radiation-Seeded Cascades}

Curvature radiation (CR), arising from charges following curved field lines, was the original mechanism proposed for generating pair-creating photons in the pulsar magnetosphere (Sturrock 1971; Ruderman \& Sutherland 1975), and is still commonly invoked today.

The spectrum of photons from a relativistic charge moving along a curved path is formally identical to a synchrotron spectral shape, but with the field lines' radius of curvature $\rho$ (and particle energy $\gamma_{b}$ ) determining the spectrum's characteristic energy $\varepsilon_{c}=\hbar \omega_{c} /\left(m c^{2}\right)=3 c \hbar \gamma_{b}^{3} /\left(2 \rho m c^{2}\right)$. The instantaneous spectrum of curvature radiation is thus easily found from the standard synchrotron power formula, with the above substitution:

$$
\frac{d N(\varepsilon)}{d t}=\frac{\sqrt{3}}{2 \pi} \frac{\alpha c}{\rho} \frac{\gamma_{b}}{\varepsilon} F\left(\frac{\varepsilon}{\varepsilon_{c}}\right)
$$


where $F(x)=x \int_{x}^{\infty} K_{5 / 3}(t) d t, K_{5 / 3}$ is a modified Bessel function of the second kind, and $\alpha$ is the electromagnetic fine-structure constant. The function $F\left(\varepsilon / \varepsilon_{c}\right)$ peaks at $0.29 \varepsilon_{c}$, and falls off at higher energies as $e^{-\varepsilon / \varepsilon_{c}}$.

The total spectrum emitted by a primary charge as it loses all of its energy will be the integral of (12) over time, as $\gamma_{b}$ decays. There is some model-dependent ambiguity in doing so, however: the accelerating E field may still be present as curvature radiation is happening, so $\gamma_{b}$ may be subject to replenishment. Furthermore, integrating equation (12) over $\gamma_{b}$ is numerically somewhat tedious. However, we note two useful approximations. All of the high-energy photons are emitted early in the particle's life, before it has escaped the pair formation region. In addition, the high-energy end of the integrated spectrum is very similar in shape to the high-energy end of the spectrum in (12).

With these in mind, we may approximate the curvature spectrum integrated over a short time by the instantaneous spectrum in (12), normalized to the energy emitted over a short time. For overall normalization, we chose to let the beam particles radiate over a time of $1 \mathrm{~km} / c$, typical of acceleration regions in polar cap models. For all but one of our composite curvature simulations, this is a short enough time that only a small fraction of $\gamma_{b}$ is lost, and the instantaneous shape of (12) is nearly identical to the integrated spectrum accounting for decrementing $\gamma_{b}$ over time accordingly. (The exception is our energy-boosted $\gamma_{b}=5 \times 10^{6}$ run, for which beam replenishment is necessary to keep radiation at constant power over this distance.) We therefore used as a curvature seed spectrum

$$
N_{\mathrm{CR}}(\varepsilon)=\frac{\sqrt{3}}{2 \pi} \frac{(1 \mathrm{~km})}{\rho} \frac{\gamma_{b} \alpha}{\varepsilon} F\left(\frac{\varepsilon}{\varepsilon_{c}}\right)
$$

Since this is a pair cascade simulation, we need to choose curvature parameters $\gamma_{b}$ and $\rho$ that will lead to pair production. (We remind the reader that our monoenergetic calculations treat a simple dipolar magnetic field; the parameter $\rho$ does not need to be specified there.) If we choose values typical of standard models of the polar flux tube (e.g., Cheng \& Ruderman 1977), we find that CR cannot easily produce photons energetic enough to generate a cascade. We carried out simulations with $\rho=100 \mathrm{~km}$ (an appropriate radius of curvature for magnetic dipole field lines), and found that $\gamma_{b} \gtrsim 10^{7}$ is needed in order to overcome the opacity limit and allow significant pair creation.

Such high beam energies can be hard to achieve in the standard models; pair cascades are difficult to initiate in those models (e.g., Weatherall \& Eilek 1997). Some authors have attempted to get around this

problem by invoking highly curved field configurations. We wished to follow this tradition, but without specifying a specific (unmotivated) non-dipolar field geometry. We therefore performed a second set of curvature spectrum seeded runs, boosting the characteristic curvature energy $\varepsilon_{c}$ by a factor of 10 by lowering $\rho$ to $10 \mathrm{~km}$ in equation (13). (Boosting curvature radiation in this manner is frequently done in the literature to encourage a pair cascade; however, we should emphasize that we only boosted the photon spectrum and did not change the field configuration.) These curvature seeded runs are referred to as the 'energy-boosted' runs in what follows.

\subsection{Magnetic Resonant Inverse Compton-Seeded Cascades}

Inverse Compton scattering (ICS) of ambient thermal photons by a primary particle beam has recently been gaining favor as a cascade seed mechanism (e.g. Daugherty \& Harding 1986; Kundt \& Schaaf 1993; Sturner \& Dermer 1994; Harding \& Muslimov 1998). In the strong pulsar magnetic field, the scattering 
process happens with greatly increased cross-section at resonances of the cyclotron frequency (Daugherty \& Harding 1986; Dermer 1990; Chang 1995). If ICS is an important process in the magnetosphere, radiation losses may limit primary beam energies (to $\gamma_{b} \sim 10^{4}$ ) while producing photons capable of pair creation (Sturner 1995). Indeed, when ICS losses are significant, beam energies may well never approach those necessary for energetic CR.

The lowest, and most important resonant interaction (Daugherty \& Harding 1986) happens when the unscattered photon frequency $\omega$ equals the cyclotron frequency $\omega_{B}$ (or $\left.\epsilon=\epsilon_{B}=\hbar \omega_{B} / m c^{2}=B / B_{c r}\right)$ in the particle's rest frame. Relativistic kinematics then gives for the scattered photon energy $\varepsilon_{s}=\epsilon_{B} / \gamma_{b}(1-\beta \cos \theta)$ in the stellar frame, where $\theta$ is the angle the initial photon's direction makes with $\mathbf{B}$. The absolute limits on the scattered energy are therefore at the extremes of $\cos \theta: \epsilon_{B} /\left(2 \gamma_{b}\right) \leq \varepsilon \leq 2 \gamma_{b} \epsilon_{B}$. When $\gamma_{b}$ is large and all photon angles are present, this represents a large frequency range into which photons are scattered by magnetic resonant ICS.

The seed photon spectrum resulting from ICS depends upon the ambient photons present (such as thermal photons above the polar cap), and so cannot easily be calculated analytically. We rely, therefore, on numerical simulation of magnetic ICS photon spectra from various source beams, from Daugherty \& Harding (1989; DH89). Their figure 9 shows the total spectrum for magnetic resonant ICS from a monoenergetic primary beam. It shows an approximate power-law of slope $\sim-1.2$ in photon number versus energy. The power-law seems to extend to very low energies, and cuts off sharply at about $2 \gamma_{b} \epsilon_{B}$, which is the absolute limit derived above. Although they considered only a somewhat low-energy beam $\left(\gamma_{b}=200\right)$, we extrapolated their results to beams of higher energy without modification.

We therefore assumed a magnetic ICS spectrum

$$
N_{\mathrm{ICS}}(\varepsilon)=\frac{0.8 \gamma_{b} m c^{2}}{\varepsilon_{\max }^{0.8}} \varepsilon^{-1.2}, 0<\varepsilon<\varepsilon_{\max }
$$

The cutoff energy was related to the Lorentz factor of the primary beam by $\varepsilon_{\max }=2 \gamma_{b} \epsilon_{B}$. The normalization was chosen to give complete energy loss of the primary beam into scattered photons.

\subsection{Results of Composite Simulations}

We computed composite CR-seeded cascades for primary beam energies in the range $10^{6} \leq \gamma_{b} \leq 10^{7}$, and with seed photons corresponding to two different curvature radii ( $\rho=100 \mathrm{~km}$ and $\rho=10 \mathrm{~km}$ ), corresponding to regular and 'energy-boosted' curvature spectra, respectively. (As described above, a pure dipolar field was used in all simulations; our choices of $\rho$ are significant only as a parameter in the curvature emission spectrum they define.) We also computed ICS seeded-cascades for lower primary beam energies, in the range $10^{3} \leq \gamma_{b} \leq 5 \times 10^{5}$. In all cases we computed cascades for magnetic fields $B_{*}=10^{12}$ and $10^{13} \mathrm{G}$. Very high beam energies for $B_{*}=10^{13}$ were not simulated since equation (7) no longer provided a good fit to the necessary high- $\varepsilon$ monoenergetic DFs there.

\subsubsection{Efficiencies and Multiplicities}

Some of the more important cascade quantities are the fractions of the primary beam's power which go into cascade-produced leptons and into energetic photons, and the overall numbers of each created. We present these results in Tables 3 (for leptons) and 4 (for photons), and recall that these quantities are 
defined per primary beam particle (in contrast with the monoenergetic efficiencies, which were defined per seed photon). Formulae used to define and calculate these quantities were provided in equations (10a) and (10b).

Table 3 summarizes the overall lepton number multiplicity $M^{l}$ and lepton fractional power $P^{l}$ for all our composite runs. We see that the lepton multiplicity and fractional power always increase with beam energy. The high-field case showed much better energy conversion than the low-field case; this is due to both the higher probability of pair creation and to the fewer synchrotron photons created during cascades in strong fields. For the $B_{*}=10^{12}$ magnetic ICS computations, no cascade develops for beam energies below $\gamma_{b}=2 \times 10^{4}$. In general, lepton multiplicities were much higher for the most energetic curvature-seeded cascades than for any ICS-seeded cascades, but the ICS cascades generally had better energy conversion efficiencies.

Table 4 summarizes energetic photon multiplicities and fractional power values for all of our composite runs. The general results seen in the lepton table are also seen here, although there are a few differences. The photon multiplicity $M^{p}$ generally increases with $\gamma_{b}$, except for CR-seeded cascades in the $10^{13} \mathrm{G}$ field. Photon fractional power $P^{p}$ nearly always increases with $\gamma_{b}$; again, the exceptions are for CR in the $10^{13}$ G field. Comparing Tables 3 and 4, we also note that for $B_{*}=10^{12} \mathrm{G}, M^{p} \gg M^{l}$, due to the large number of lower-energy synchrotron photons generated. At $B_{*}=10^{13} \mathrm{G}, M^{p}<M^{l}$ for ICS-seeded and high-energy CR-seeded cascades, due to the dearth of secondary photons in these one-generation cascades. At low primary beam energies, CR-seeded cascades produce many photons which do not pair create, so $M^{p}$ is still large. We note that the fractional powers do not generally add to unity. Any deficit is due to synchrotron photons with energies $\epsilon<2$. The excess power in the energy-boosted $\gamma_{b}=5 \times 10^{6}$ run requires beam replenishment, and is due to our choosing consistent normalization for $N_{\mathrm{CR}}$ throughout; see discussion preceding equation (13) for this.

\subsubsection{Lepton Distribution Functions}

One of the primary reasons for this work was to find the plasma DFs predicted by pair cascade models. Despite the enormous range in cascade parameters and pair creation efficiencies encountered, we found that the composite DFs have remarkably similar shapes. Figure 8 shows some sample curvature-seeded and ICSseeded plasma DFs, sorted by magnetic field strength and seed mechanism. Normalizations are as discussed above, except for the DF which was multiplied up by the factor shown for visibility. Only the energy-boosted curvature composites are shown; the results for the standard curvature-seeded runs are qualitatively similar (but with lower efficiencies, as shown in Table 3). We see that both $B_{*}$ and the photon seed spectrum do have some effect on the resultant plasma DFs.

For $B_{*}=10^{13}$, the DFs all have a low- $p_{\|}$gap below about $20 m c$; no slow leptons are present. This is consistent with the narrower 'kernel' DF widths for $B_{*}=10^{13} \mathrm{G}$, since low-energy photons do not pair create at the small $\left(1 / \gamma_{b}\right)$ angles we considered here. In contrast, the $B_{*}=10^{12}$ DFs all extend down to $p_{\|}<.01$. Synchrotron emission, significant in lower fields, helps to achieve this in two ways. First, leptons which are born in excited Landau levels lose energy by synchrotron radiation, and although $\beta$ is roughly conserved along $\mathbf{B}$ the leptons still lose $p_{\|}$as their total $\gamma$ decreases. Second, synchrotron photons which themselves create pairs are typically at lower energies and larger angles with $\mathbf{B}$ than the primary beam photons, so the leptons are born with lower $p_{\|}$to begin with (and may still lose $p_{\|}$by the first mechanism).

The curvature-seeded DFs all peak somewhere between $p_{\|}=10$ and 50 for CR cascades. The low end 

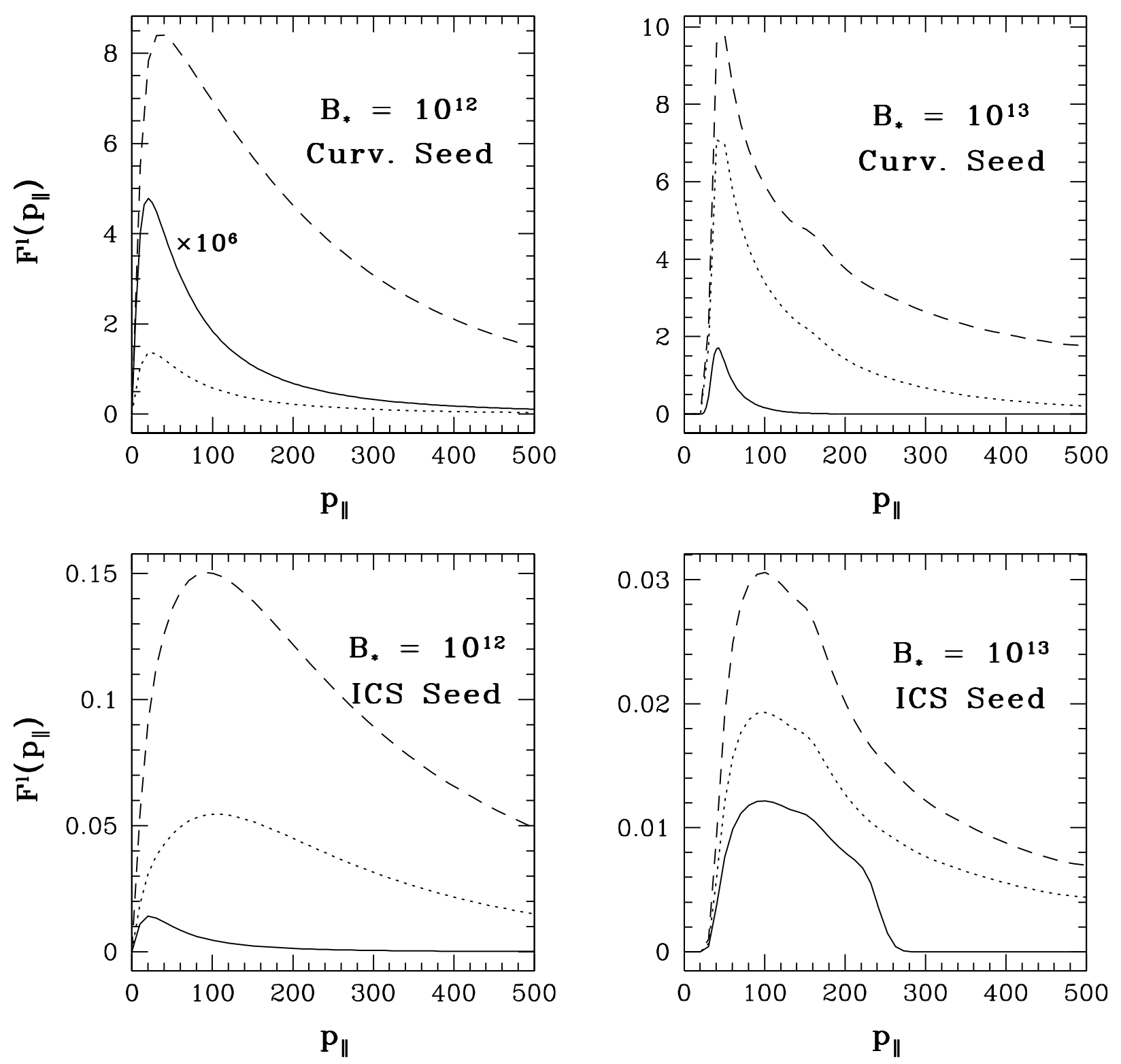

Fig. 8.- Composite Lepton DFs: Stellar Frame. Sample DFs are given for pair plasmas arising from both curvature and magnetic resonant inverse Compton seed photon spectra. The DFs are shown as they appear in the (corotating) star frame. The curvature-seeded runs shown here are all from the 'energy-boosted' runs, and beam energies of 1,2 and $5 \times 10^{6} \mathrm{mc}^{2}$, corresponding to the solid, dashed, and dotted lines, respectively. For the $B_{*}=10^{12}$ ICS runs, the respective line types are for beam energies of 2,10 , and $50 \times 10^{4}$. For the $B_{*}=10^{13}$ ICS runs, beam energies are $10^{3}, 10^{4}$, and $10^{5}$, respectively. Normalizations are as discussed in $\S 4$ of the text. Note the low-momentum cutoffs of the high-field DFs. The $B_{*}=10^{12}, \gamma_{b}=10^{6}$ curvature DF has been scaled up by the factor shown for visibility. 
has a very strong cutoff below $p_{\|}=10$, while the DF drops off slightly more gradually at high $p_{\|}$. The ICS-seeded DFs all peak at about $p_{\|}=100$, except for the $B_{*}=10^{12}, \gamma_{b}=10^{3}$ DF which peaks at $\sim 20 \mathrm{mc}$. Generally, the ICS DFs drop off more gradually than the CR DFs at large $p_{\|}$. The $B_{*}=10^{13}$ ICS DFs mimic the seed spectrum power-law at high momenta (consistent with the narrow monoenergetic 'kernel' DFs for the strong field).

The results simplify if the DFs are transformed into the center-of-momentum (CM) frame of the plasma. To do this, we numerically found the average Lorentz factor $\bar{\gamma}$ and average momentum $\overline{p_{\|}}$of the DF; then the transformation velocity is given by $\beta_{\mathrm{CM}}=\overline{p_{\|}} / \bar{\gamma}$. Once this is done, most of the DFs are found to have shapes that are remarkably close to a thermal distribution, which in this context means a 1-D relativistic Maxwellian (Jüttner - Synge) distribution:

$$
f\left(p_{\|}\right)=\frac{1}{2 K_{1}(\zeta)} \exp \left[-\zeta \gamma\left(p_{\|}\right)\right]
$$

where $\zeta=m c^{2} / k_{B} T$ is the inverse temperature of the distribution, $K_{1}$ is the modified Bessel function, and $\gamma\left(p_{\|}\right)=\sqrt{1+p_{\|}^{2}}$.

Figure 9 shows the DFs in their respective CM frames. These DFs have all been normalized to $\int f\left(p_{\|}\right) d p_{\|}=1$. The DFs for $B_{*}=10^{12} \mathrm{G}$ are nearly indistinguishable from their corresponding best fits (which we do not show) to equation (13), while the higher field DFs show asymmetries not present in the Maxwellian fits. Also, the DFs for $B_{*}=10^{12}$ have nearly identical shapes (temperatures) when seen in their CM frames, while the $B_{*}=10^{13}$ DFs show temperature dependence with beam energy.

We present statistics of the DFs in Table 5. Best-fit parameters of equation (15) are given, along with the CM frame transformation Lorentz factors $\gamma_{\mathrm{CM}}=\left(1-\beta_{\mathrm{CM}}^{2}\right)^{-1 / 2}$. Values of $\gamma_{\mathrm{CM}}$ ranged from $38-890$, showing that the secondary plasma is indeed moving relativistically outward. However, values of the inverse temperature $\zeta$ show that the plasma is only mildly relativistic in its own frame, with $k_{B} T \sim m c^{2}$ for all the low-field cascades, and $k_{B} T \sim$ several $m c^{2}$ for high beam energies and cooler at lower beam energies in the high-field cascades. Even when $\bar{\gamma}$ was much larger than $\gamma_{\mathrm{CM}}$, the intrinsic plasma temperature is relatively low, and the apparent width of the DF in the stellar frame is due to the Lorentz boost when passing to that frame (see also Weatherall 1994).

\subsubsection{Photon Spectra}

We also generated photon spectra for the composite simulations. Figure 10 shows the photon spectra for the energy-boosted curvature seeded and the ICS seeded cascades. The relative heights of each spectrum correspond directly to the relative normalizations for the cascade seeds. The most important trend to note is that the lower-field runs have steep photon spectra for lower-energy $\gamma$-rays (for the largest $\gamma_{b}$ values), while the high-field runs have flat spectra. This directly reflects the production, or not, of abundant synchrotron photons in a multi-step cascade.

The photon spectra for the $B_{*}=10^{12}$ field include both synchrotron photons generated in the cascade, and primary photons which escaped the magnetosphere without producing a lepton pair. The synchrotron contributions are shown separately (as the dotted lines) alongside the total spectra in figure 10. No secondary high-energy photons were generated in the $B_{*}=10^{13}$ runs that did not themselves pair produce, so the only photons escaping from the cascade are lower-energy seed photons which did not pair create.

For both CR and ICS cascades, at low beam energies, the photon spectra are independent of $B_{*}$. Each 

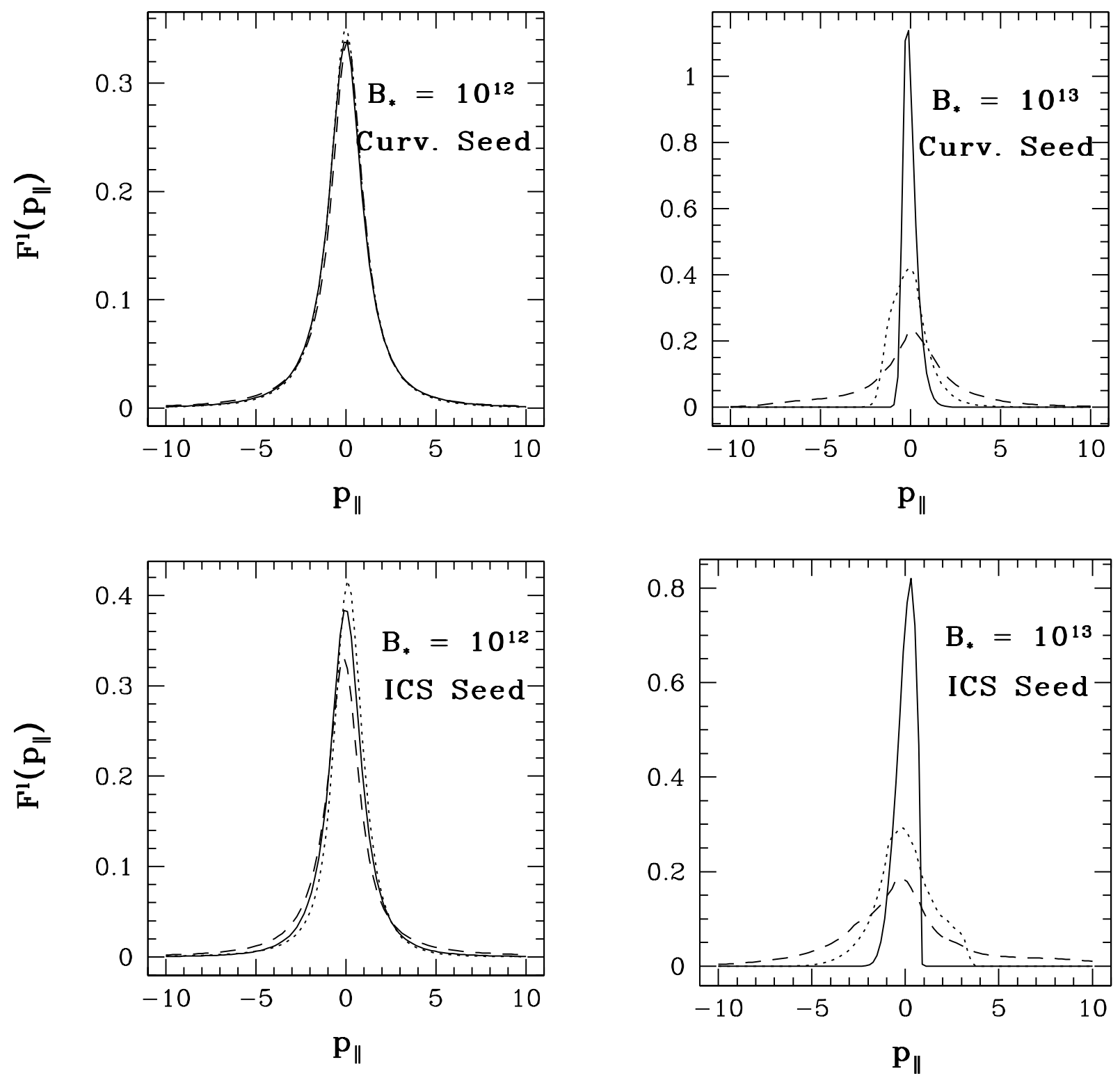

Fig. 9.- Composite Lepton DFs: CM frame. The DFs have been transformed to their center-of-momentum frames, and have been normalized to 1 . Beam energies are associated to line types as in figure 8 . Statistics of the CM transformation and Maxwellian fit parameters in the CM frame are given in Table 5. 

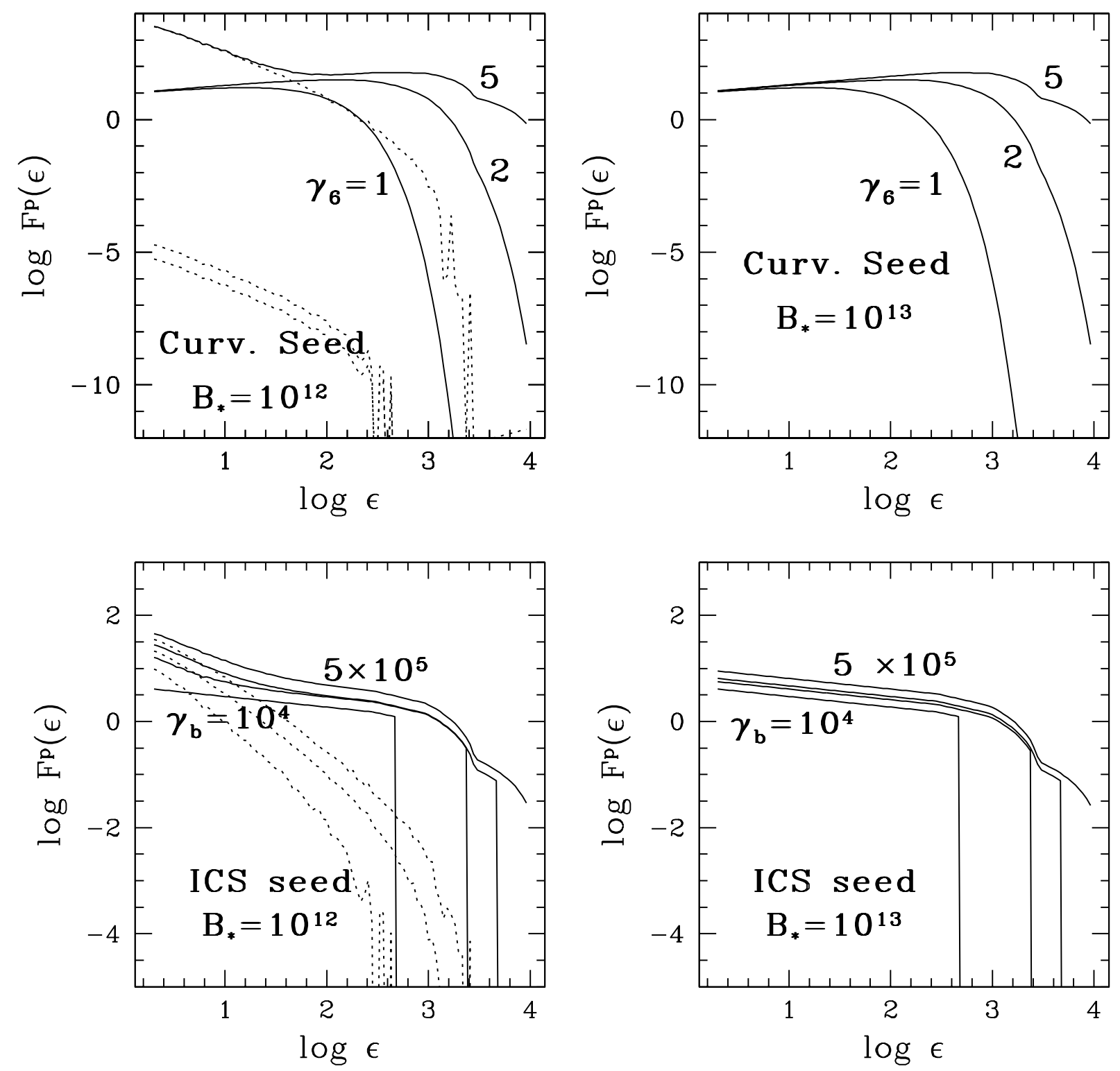

Fig. 10. - Composite Photon Spectra. Spectra are given for $\gamma$-rays of $\epsilon>2$, from curvature and ICS seeded pair cascades. The curvature-seeded runs were from our set with 'energy-boosted' seed photons. For the CR-seeded spectra, $\gamma_{6}$ refers to the primary beam Lorentz factor divided by $10^{6}$. Normalizations are as discussed in $\S 4$ of the text. Both the leftover seed photons and cascade-generated synchrotron photons are added together for the spectra drawn with solid lines, while dotted lines are the spectra from highergeneration synchrotron photons alone. No secondary photons are generated at these beam energies (assuming $1 / \gamma_{b}$ beaming) in the $10^{13} \mathrm{G}$ case, so only the seed photons which did not produce pairs are shown. Note that the vertical axis for curvature cascades is twice the scale of the ICS cascades. 
spectrum is just the primary photon spectrum, depleted by those photons which created pairs. Synchrotron photons (which exist only for the $B_{*}=10^{12} \mathrm{G}$ composites) are not numerous enough to influence the photon spectral shape at low beam energies. It is evident that the synchrotron photons decide the low- $\epsilon$ steep spectral shape at large beam energies in the $10^{12} \mathrm{G}$ field. There is a high-energy break above which the residual primary spectrum is seen again, due to the steepness of the high-energy cutoff of the secondaries.

\section{DISCUSSION AND SUMMARY}

We have simulated the pair production cascade which may occur in pulsar magnetospheres. We did this by means of a numerical code which tracks leptons and photons as they propagate upward above the magnetic polar cap of a rotating neutron star with a dipolar magnetic field. The code simulates magnetic pair production by the photons and quantized synchrotron emission by the leptons, beginning with a population of monoenergetic seed photons. We ran simulations with a range of seed energies, angles, and magnetic field strengths to obtain detailed results over a substantial parameter range relevant to pair cascade models. We used these results as kernels to model pair cascades produced by an initial particle beam, either by curvature radiation or by magnetic resonant inverse Compton scattering.

\subsection{Our Results}

Our main results can be summarized succinctly.

Cascade Onset and Development. We find that $B \sim 10^{11} \mathrm{G}$, or larger, is required in order for the cascade to go if standard values are assumed for the primary beam energies. This is a consequence of the opacity condition, (6), and is independent of the magnetic field geometry. This suggests that slow rotation-powered pulsars, and low-field millisecond pulsars, either have no pair production or have seed photon energies much higher than the standard models predict. When the cascade occurs, we find that it develops and ends spatially within $\delta r<r_{*}$ of the creation of the photon seeds, thus temporally within several microseconds after each photon injection event. In an actual pulsar magnetosphere, it is possible that the seed photons are injected continuously, but gap models with rapid temporal variability of the accelerating potential and sparking (Ruderman \& Sutherland 1975) are perhaps more likely due to the nonlinearity and magnitude of gap accelerating potentials. If sparking occurs, the cascade timescale seen here may be directly connected to $\mu$ sec flickering known as microstructure.

Efficiencies and Multiplicities. Our computed efficiencies and multiplicities stand in contrast to assumptions made in the literature. In particular, it is difficult to transfer a large fraction of the primary beam's energy to the pair plasma; some, often most, of it escapes as photons. In addition, our computed multiplicities (number of pairs produced per primary beam particle) are often small, only a few tens. Only rarely do they reach the large values, $>10^{3}$, which are often assumed in the literature; curvature radiation with a large beam energy seems to accomplish this best.

The Pair Plasma. We find that the plasma DFs in all cases can be well described as relativistic Maxwellians in the plasma's comoving frame. They generally have temperature $k T \sim m c^{2}$. The plasma flows out along field lines with Lorentz factors $\gamma_{\mathrm{CM}} \sim 100-1000$. The resulting Lorentz boost into the pulsar frame gives the characteristic, asymmetric shape seen in figure 8.

The Escaping Photons. We find that the escaping photon spectra tend to be steep in lower magnetic 
fields, due to the abundance of cooler, secondary synchrotron photons. Conversely, they tend to be flatter in higher fields, where synchrotron photons are rare and the spectra are dominated by those seed photons which escaped pair creation.

In addition to these general trends, the details of the cascade development are sensitive to the initial field strength and also the seed photon spectrum.

Effect of Magnetic Field Strength. Qualitative differences in the pair cascade occur for initial magnetic field strengths of $B_{*}=10^{13}$ compared to $B_{*}=10^{12} \mathrm{G}$. The 'transition' field strength is likely near $B_{*}=0.1 B_{c r} \sim 4.4 \times 10^{12} \mathrm{G}$. The differences are due to the abundance, or dearth, of secondary synchrotron photons. Below this transition field strength, leptons tend to be born into excited Landau levels and copious synchrotron emission takes place. This occurs because the pair formation events tend to be governed by opacity rather than energetics. Above this transition field, pair formation events are generally controlled by energetics, so that leptons are usually born in their lowest Landau state, and synchrotron emission is rare or absent.

Effect of Seed Photon Source. The overall cascade efficiency is sensitive to the seed mechanism. We find that CR-seeded models give pair multiplicities of up to a few times $10^{3}$, but typically do not transfer much of the beam energy to the pair plasma. In contrast, ICS-seed models give multiplicities of fewer than 100 , but can efficiently transfer the beam energy into the pair plasma (however, this requires ambient thermal photons at the magnetic resonance of the beam, which we assumed at the outset). Furthermore, the ICS mechanism operates at much lower beam energies than does curvature radiation.

Independence of Seed Photon Source. Despite the enormous range of cascade efficiencies, we find that the plasma and photon distributions are fairly similar for both seed photon spectra which we used. Perhaps the likenesses should not be too surprising, since the general ingredients required for a pair creation cascade are somewhat independent of seed mechanism. First, photons must be of sufficient energy to pair produce; the lower-end shape of the photon spectrum does not affect the plasma DFs at all. We chose photon seed spectra based on typical beam energies in the literature; only a small fraction of the seed photons these charges produce are able to seed the cascade. Second, a high-energy cutoff is always present in the photon spectrum. This cutoff, together with the low-energy cutoff where the primary photons no longer produce pairs, limits the effective (pair-producing) seed photon spectrum to a relatively narrow range of energies. Thus, many qualitative features of the pair cascade are not very sensitive to the mechanism of radiation which seeds the cascade (provided that it seeds a cascade at all).

\subsection{Comparison to Other Simulations}

Other authors have performed numerical pair cascade simulations; however, the focus has usually been on the final escaping $\gamma$-rays rather than on the pair plasma.

DH82 simulated cascades seeded by curvature radiation. Our CR-seeded photon spectra for high beam energies and field strengths $B_{*}=10^{12} \mathrm{G}$ agree qualitatively with those of DH82, but we find much softer spectra in the higher $B_{*}$ case than they do. The single lepton DF shown in DH82 (their figure 7) is for $B_{*}=10^{12} \mathrm{G}$, and a beam energy of $10^{13} \mathrm{eV}\left(\gamma_{b} \sim 2 \times 10^{7}\right)$. It shows a rough power-law above $p_{\|} \sim 100 \mathrm{mc}$, and few pairs at lower momenta. In addition, they predict that more low- $p_{\|}$pairs will be created in higher fields, since photons of lower energy will be opaque to pair creation. We believe that their conclusions (in contrast with what we present here) can ultimately be traced to their assumption that both members of the 
pair share the direction and half the energy of the parent photon. (Note that DH82 predates Daugherty \& Harding 1983, where they present energy-differential pair production cross-sections. In stronger fields, the lack of synchrotron radiation is more significant than the ability of lower-energy photons to pair create, and so the DFs still have fewer leptons at low $p_{\|}$.)

Sturner et al. (1995) investigated cascades seeded by inverse Compton radiation. They do not present the final plasma DFs at all. Our ICS-seeded photon spectra for $B_{*}=10^{12}$ agree qualtitatively with their results for $B_{*}=4 \times 10^{12}$. Note that we find much flatter spectra for our $B_{*}=10^{13}$ simulations (for which Sturner et al. did not offer spectra).

\subsection{Comparison to other Plasma DFs}

The plasma DF is critical in determining the wavemodes the plasma can support. Knowledge of the DF is therefore crucial to calculations of radio emission and signal propagation in the pulsar magnetosphere. Previous work has had to assume some some analytic expression, not necessarily physically motivated. Some work has chosen analytically convenient forms, such as cold plasma (delta function), boxcar and bell-curve DFs, and relativistic Maxwellians (both in the comoving and pulsar frames). Our work supports one of these assumptions, namely, a comoving Maxwellian. Our final DFs are only marginally relativistically hot, with temperatures $k_{B} T \sim m c^{2}$ in most cases, and lower temperatures in $B_{*}=10^{13}$ fields when the beam energy is just above the threshold to seed a pair cascade. The large spread in $p_{\|}$in the pulsar-frame DFs is simply due to of Lorentz boosting.

In addition, some authors have used an analytic representation of a DF which Arons (1981) presented as a cartoon model. The Arons cartoon-DF has a peak at $p_{\|} \sim$ a few $m c$. It drops off exponentially above $p_{\|} \sim 10^{3}-10^{4}$. Both of these features are roughly consistent with our lower-field results, where synchrotron photons are produced. Our higher-field results have a qualitatively similar shape but at higher $p_{\|}$values. In addition, the Arons cartoon-DF has a flat region between these extremes. We do not see this feature in our DFs, even in the $B_{*}=10^{12}$ case.

\subsection{Impact on Future Work}

We anticipate that the results presented here will be most relevant to plasma-based modeling of the magnetosphere and of the radio emission mechanisms. We give two examples.

The pair plasma plays a critical role in the electrodynamics of the polar flux tube; it is often assumed to short out the parallel electric field and terminate the acceleration region. Ruderman \& Sutherland (1975) estimated that the pair multiplicity should be around $\gamma_{b} / \gamma_{\text {pairs. }}$. This is typically two orders of magnitude larger than the multiplicities we find (except for the high-field magnetic ICS runs). Shibata et al. (1998) agree with Ruderman \& Sutherland that these large multiplicities $\left(10^{3}-10^{5}\right)$ are necessary to short out the accelerating $\mathbf{E}_{\|}$field. Our results suggest that the situation is not so simple. If complete shorting-out cannot be maintained, we might expect a complex, non-steady plasma flow in the region.

Instabilities in the pair plasma are often assumed to give rise to coherent radio emission. One such mechanism is a two-stream instability, driven by relative motion of the two signs of charge (e.g., Buschauer \& Benford 1976). The high temperatures assumed by some authors tend to suppress this instability (e.g., Weatherall 1994); our results however find a lower temperature which may be favorable for this mechanism. 
We are presently investigating the details of this instability in the pair plasmas found in our calculations (Arendt \& Weatherall, in progress).

\section{ACKNOWLEDGEMENTS}

We would like to thank Galen Gisler, who helped formulate the basic calculation and set up the initial code. Many helpful discussions were had with members of the New Mexico Tech pulsar group, including Tim Hankins, Jim Weatherall, David Moffett, Jeff Kern, and Tracey DeLaney. J.E. would also like to thank Jon Arons and Don Melrose for useful discussions. An anonymous referee provided some useful suggestions, as well. We are grateful for partial funding that was provided by NSF grants AST-9315285 and AST-9618408.

\section{APPENDIX: CODE DETAILS}

We present here some of the relevant physical and computational details of our pair cascade code. We discuss the kinematics of the pair production process, and its implementation in our code. We also present the relevant details of synchrotron photon production.

The probability for photon pair production can be described by an attenuation coefficient (the inverse of the mean free path) $R(\chi)$, where $\chi$ was defined above equation (2). The (spin and polarization averaged) differential attenuation coefficient is given (in the frame moving along $\mathbf{B}$ satisfying $\mathbf{k} \cdot \mathbf{B}=0$ ) in Daugherty \& Harding (1983) as

$$
\frac{d R(\kappa, \chi)}{d \kappa} \approx \frac{2 \alpha}{\sqrt{27} \lambda_{C}} \frac{B}{B_{c r}} \frac{2+\kappa(1-\kappa)}{\chi \kappa(1-\kappa)} K_{2 / 3}\left[\frac{1}{3 \chi \kappa(1-\kappa)}\right]
$$

where $\alpha$ is the fine structure constant, $\lambda_{C}=\hbar / m c, \kappa$ is the fraction of photon energy given to one member of the created pair, and $K_{2 / 3}$ is the modified Bessel function. The behavior of $d R / d \kappa$ with $\kappa$ determines how a given photon distributes its excess (above $\epsilon=2$ ) energy between the electron and positron.

We created a grid of numerically determined values of $d R / d \kappa$ over a range of $\chi$ and $\kappa$, and used interpolation when needing $d R / d \kappa$ in the code. To find the total attenuation coefficient $R(\chi)$, we numerically integrated $d R / d \kappa$ over $\kappa$ for each fixed value of $\chi$. The total $R(\chi)$ was then used to determine when to create a pair. At each timestep, we multiplied the distance traveled by each photon by the $R(\chi)$ appropriate to the photon's environment, and added this 'fractional mean free path' to the photon's optical depth $\tau$, approximating

$$
\tau=\int R(\chi) d s
$$

After $\tau>1$, we created a lepton pair in place of the photon. To find the split in energy amongst the members of the pair, we needed partial probabilities as well. We integrated $d R / d \kappa$ from 0 to $\kappa$ for a set of values of $\kappa$ and normalized by $R(\chi)$. This function allowed us to use a random number to choose (with interpolation) $\kappa$ for a given pair production event.

Energy and momentum along $\mathbf{B}$ are conserved in one-photon magnetic pair production. Momentum of the particles and photons across $\mathbf{B}$ is not conserved; the magnetic field provides for the difference, which allows the process to occur kinematically. Once we determined the fractional energy $\kappa$ given to one lepton, we chose a random number uniform in $\cos ^{2} \theta$ (over the kinematically allowed angles) to determine its pitch angle (in the $\mathbf{k} \cdot \mathbf{B}=0$ frame). The other lepton's energy and pitch angle were then simply determined by the conservation laws. 
We also required an expression for the synchrotron emissivity of an electron or positron. We needed both the differential emissivity as a function of emitted photon energy (to decide how energetic a photon to make), and total synchrotron emissivity over the interesting photon range $(\epsilon>2)$. Harding \& Preece 1987 give a useful approximation to the differential synchrotron emissivity of a lepton of energy $\gamma$, in a frame moving along $\mathbf{B}$ such that the lepton's momentum parallel to $\mathbf{B}$ vanishes. We divided this by the photon energy $\epsilon$ to get the differential photon number production rate in a magnetic field of strength $B$ :

$$
\frac{d \dot{N}(\gamma, \epsilon, B)}{d \epsilon}=\frac{\sqrt{3}}{2 \pi} \alpha \omega_{B} \frac{f}{\epsilon}\left[y \int_{y}^{\infty} K_{5 / 3}(x) d x+y^{3} f\left(\frac{3 \gamma B}{2 B_{c r}}\right)^{2} K_{2 / 3}(y)\right]
$$

where $y \equiv 2 \epsilon B_{c r} /\left(3 f \gamma^{2} B\right)$, and $f \equiv 1-\epsilon / \gamma$ is the fraction of lepton energy remaining after the photon emission.

The code created a synchrotron photon at the lepton's location when the lepton's total energy would have been lost if it radiated its energy away linearly at its present rate, defining a sort of 'optical depth' for the lepton's momentum transverse to the field. We then decrement this opacity by the fraction of actual energy radiated in the photon creation event, and continue the process until no more photons with $\epsilon>2$ can be created by synchrotron radiation. After that, the classical synchrotron power formula is used on the lepton to decrement its energy with time. This approximate method was agrees well with the classical synchrotron loss rate.

Once the decision is made to create a synchrotron photon, we use a grid of $d \dot{N} / d \epsilon$ integrated from 0 to $\epsilon$ on a set of parameter values to determine the photon's energy, using a similar algorithm to the one described above for pair production.

\section{REFERENCES}

Arons, J., \& Scharlemann, E. T. 1979, ApJ, 231, 854

Arons, J. 1981, in Proc. Varenna Summer School and Workshop on Plasma Astrophysics, ed. T. D. Guyenne \& G. Lévy (Noordwijk: ESA), 273

Arons, J. 1983, ApJ, 266, 215

Arons, J., \& Barnard, J. J. 1986, ApJ, 302, 120

Arons, J. 1992, in IAU Colloquium No. 128, ed. T. H. Hankins, J. M. Rankin, \& J. A. Gil (Zielona Góra, Poland: Pedagogical Univ. Press), 56

Beskin, V. S., Gurevich, A. V., \& Istomin, Ya. N. 1988, Astrophys. Space Sci., 146, 205

Beskin, V. S., Gurevich, A. V., \& Istomin, Ya. N. 1993, Physics of the Pulsar Magnetosphere (Cambridge: CUP)

Buschauer, R. \& Benford, G. 1977, MNRAS, 179, 99

Bussard, R. W., Alexander, S. B., \& Mészáros, P. 1986, Phys. Rev. D, 34, 440

Chang, H.-K. 1995, Astron. Astrophys., 301, 456

Cheng, A. F., \& Ruderman, M. A. 1977, ApJ, 214, 598

Daugherty, J. K., \& Harding, A. K. 1982, ApJ, 252, 337

Daugherty, J. K., \& Harding, A. K. 1983, ApJ, 273, 761 
Daugherty, J. K., \& Harding, A. K. 1986, ApJ, 309, 362

Daugherty, J. K., \& Harding, A. K. 1989, ApJ, 336, 861

Daugherty, J. K., \& Lerche, I. 1975, Ap. Space Sci., 38, 437

Dermer, C. D. 1990, ApJ, 360, 197

Gedalin, M., Melrose, D. B., \& Gruman, E. 1998, Phys. Rev. E, 57, 3399

Goldreich, P. \& Julian, W. H., 1969, ApJ, 157, 869

Hankins, T. H., in Pulsars: Problems and Progress (IAU Colloquium 160), ed. S. Johnston, M. A. Walker and M. Bailes (San Francisco: ASP), 197

Harding, A. K., \& Muslimov, A. G., 1998, ApJ, 508, 328

Harding, A. K., \& Preece, R. 1987, ApJ, 319, 939

Hirotani, K. \& Shibata, S., 1999, MNRAS, 308, 54

Jackson, J. D. 1975, Classical Electrodynamics (New York: John Wiley \& Sons)

Johnson, M. H., \& Lippman, B. A., 1949, Phys. Rev., 76, 828

Kazbegi, A. Z., Machabeli, G. Z., \& Melikidze, G. I. 1991, MNRAS, 253, 377

Kundt, W. \& Schaaf, R. 1993, Astrophys. Space Sci., 200, 251

Lyubarskii, Yu. E. 1996, Astron. Astrophys., 308, 809

Lyutikov, M., Blandford, R. D., \& Machabeli, G. 1999, MNRAS, 305, 338

Melrose, D. B. 1992, in IAU Colloquium No. 128, ed. T. H. Hankins et al. (Zielona Góra, Poland: Pedagogical Univ. Press), 307

Melrose, D. B. 1995, J. Astrophys. Astr., 16, 137

Rankin, J. M. 1983, ApJ, 274, 333

Romani, R. W., 1996, ApJ, 470, 469

Rudak, B. \& Dyks, J., 1999, MNRAS, 303, 477

Ruderman, M., \& Sutherland, P. 1975, ApJ, 196, 51

Shibata, S., Miyazaki, J., \& Takahara, F. 1998, MNRAS, 295, L53

Sturner, S. J. 1995, ApJ, 446, 292

Sturner, S. J. \& Dermer, C. D., 1994, ApJ, 420, L79

Sturner, S. J., Dermer, C. D., \& Michel, F. C. 1995, ApJ, 445, 736

Sturrock, P. A. 1971, ApJ, 164, 529

Tsai, W., \& Erber, T. 1974, Phys. Rev. D, 10, 492

Usov, V. V., \& Melrose, D. B. 1996, ApJ, 464, 306

Weatherall, J. C. 1994, ApJ, 428, 261

Weatherall, J. C. \& Eilek, J. A., 1997, ApJ, 474, 407

Zhang, B. \& Harding, A. K., 2000, ApJ, 532, 1150

This preprint was prepared with the AAS LATEX macros v5.0. 
Table 1. MONOENERGETIC CASCADE EFFICIENCIES: LEPTONS

\begin{tabular}{|c|c|c|c|c|c|c|}
\hline \multirow[b]{2}{*}{ Cosine Complement $\mu$} & \multicolumn{2}{|c|}{$B_{*}=10^{11} \mathrm{G}$} & \multicolumn{2}{|c|}{$B_{*}=10^{12} \mathrm{G}$} & \multicolumn{2}{|c|}{$B_{*}=10^{13} \mathrm{G}$} \\
\hline & $C^{l}$ & $E^{l}$ & $C^{l}$ & $E^{l}$ & $C^{l}$ & $E^{l}$ \\
\hline \multicolumn{7}{|c|}{$\varepsilon=300$} \\
\hline $10^{-1}$ & .83 & .029 & 3.8 & .14 & 10 & .41 \\
\hline $10^{-3}$ & $\ldots a^{a}$ & $\ldots$ & .81 & .23 & 1.5 & .48 \\
\hline $10^{-5}$ & $\ldots$ & $\ldots$ & $\ldots$ & $\ldots$ & .91 & .91 \\
\hline $10^{-7}$ & $\ldots$ & $\ldots$ & $\ldots$ & $\ldots$ & .91 & .91 \\
\hline $10^{-12}$ & $\ldots$ & $\ldots$ & $\ldots$ & $\cdots$ & .91 & .91 \\
\hline \multicolumn{7}{|c|}{$\varepsilon=1000$} \\
\hline $10^{-1}$ & 1.2 & .015 & 11. & .14 & 27. & .40 \\
\hline $10^{-3}$ & $\cdots$ & $\cdots$ & 1.2 & .13 & 4.4 & .52 \\
\hline $10^{-5}$ & $\ldots$ & $\ldots$ & .27 & .076 & 1.1 & .75 \\
\hline $10^{-7}$ & $\cdots$ & $\cdots$ & .26 & .062 & .99 & .99 \\
\hline $10^{-12}$ & $\ldots$ & $\ldots$ & .26 & .064 & .99 & .99 \\
\hline \multicolumn{7}{|c|}{$\varepsilon=3000$} \\
\hline $10^{-1}$ & 3.8 & .016 & 32. & .14 & 73. & .41 \\
\hline $10^{-3}$ & .75 & .023 & 3.5 & .13 & 12. & .52 \\
\hline $10^{-5}$ & $\ldots$ & $\ldots$ & .99 & .28 & 3.5 & .72 \\
\hline $10^{-7}$ & $\ldots$ & $\ldots$ & .92 & .29 & 1.0 & 1.0 \\
\hline $10^{-12}$ & $\ldots$ & $\ldots$ & .92 & .28 & 1.0 & 1.0 \\
\hline \multicolumn{7}{|c|}{$\varepsilon=10000$} \\
\hline $10^{-1}$ & 11. & .014 & 89. & .14 & 200 & .44 \\
\hline $10^{-3}$ & 1.2 & .013 & 11. & .14 & 35. & .57 \\
\hline $10^{-5}$ & .16 & .004 & 2.1 & .18 & 11. & .77 \\
\hline $10^{-7}$ & .10 & .002 & 1.4 & .34 & 1.5 & .78 \\
\hline $10^{-12}$ & .11 & .002 & 1.0 & .24 & 1.0 & .99 \\
\hline \multicolumn{7}{|c|}{$\varepsilon=30000$} \\
\hline $10^{-12}$ & .87 & .026 & 3.6 & .36 & 1.0 & .96 \\
\hline \multicolumn{7}{|c|}{$\varepsilon=100000$} \\
\hline $10^{-12}$ & 1.2 & .031 & 11. & .38 & 1.0 & .89 \\
\hline
\end{tabular}

${ }^{\mathrm{a}}$ No pair creation at these angles. 
Table 2. MONOENERGETIC CASCADE EFFICIENCIES: PHOTONS

\begin{tabular}{|c|c|c|c|c|c|c|}
\hline \multirow[b]{2}{*}{ Cosine Complement $\mu$} & \multicolumn{2}{|c|}{$B_{*}=10^{11} \mathrm{G}$} & \multicolumn{2}{|c|}{$B_{*}=10^{12} \mathrm{G}$} & \multicolumn{2}{|c|}{$B_{*}=10^{13} \mathrm{G}$} \\
\hline & $C^{p}$ & $E^{p}$ & $C^{p}$ & $E^{p}$ & $C^{p}$ & $E^{p}$ \\
\hline \multicolumn{7}{|c|}{$\varepsilon=300$} \\
\hline $10^{-1}$ & 390 & .998 & 24 & .569 & 15 & .366 \\
\hline $10^{-3}$ & $\ldots{ }^{\mathrm{a}}$ & $\ldots$ & 10 & .537 & 5.0 & .322 \\
\hline $10^{-5}$ & $\ldots$ & $\ldots$ & $\ldots$ & $\ldots$ & .092 & .091 \\
\hline $10^{-7}$ & $\ldots$ & $\ldots$ & $\ldots$ & $\ldots$ & .091 & .090 \\
\hline $10^{-12}$ & $\ldots$ & $\ldots$ & $\ldots$ & $\ldots$ & .098 & .098 \\
\hline \multicolumn{7}{|c|}{$\varepsilon=1000$} \\
\hline $10^{-1}$ & 55 & .833 & 73 & .580 & 42 & .353 \\
\hline $10^{-3}$ & $\ldots$ & $\ldots$ & 35 & .722 & 15 & .322 \\
\hline $10^{-5}$ & ... & .. & 9.2 & .855 & 6.2 & .030 \\
\hline $10^{-7}$ & $\cdots$ & $\ldots$ & 11 & .876 & .015 & .014 \\
\hline $10^{-12}$ & $\ldots$ & $\ldots$ & 10 & .873 & .012 & .012 \\
\hline \multicolumn{7}{|c|}{$\varepsilon=3000$} \\
\hline $10^{-1}$ & 150 & .840 & 210 & .593 & 110 & .346 \\
\hline $10^{-3}$ & 110 & .905 & 87 & .737 & 38 & .292 \\
\hline $10^{-5}$ & $\ldots$ & $\ldots$ & 32 & .485 & 5.5 & .081 \\
\hline $10^{-7}$ & $\ldots$ & $\cdots$ & 32 & .476 & 0 & 0 \\
\hline $10^{-12}$ & $\ldots$ & $\ldots$ & 33 & .486 & 0 & 0 \\
\hline \multicolumn{7}{|c|}{$\varepsilon=10000$} \\
\hline $10^{-1}$ & 470 & .849 & 630 & .614 & 320 & .386 \\
\hline $10^{-3}$ & 220 & .948 & 260 & .730 & 100 & .241 \\
\hline $10^{-5}$ & 59 & .989 & 96 & .668 & 15 & .069 \\
\hline $10^{-7}$ & 39 & .993 & 52 & .403 & .38 & .002 \\
\hline $10^{-12}$ & 42 & .992 & 51 & .409 & 0 & 0 \\
\hline \multicolumn{7}{|c|}{$\varepsilon=30000$} \\
\hline $10^{-12}$ & 370 & .944 & 140 & .350 & 0 & 0 \\
\hline \multicolumn{7}{|c|}{$\varepsilon=100000$} \\
\hline $10^{-12}$ & 580 & .937 & 420 & .298 & 0 & 0 \\
\hline
\end{tabular}

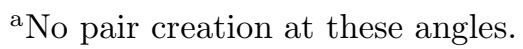


Table 3. COMPOSITE CASCADE EFFICIENCIES: LEPTONS

\begin{tabular}{|c|c|c|c|c|}
\hline \multirow[b]{2}{*}{$\gamma_{\text {beam }}$} & \multicolumn{2}{|c|}{$B_{*}=10^{12} \mathrm{G}$} & \multicolumn{2}{|c|}{$B_{*}=10^{13} \mathrm{G}$} \\
\hline & $M^{l}$ & $P^{l}$ & $M^{l}$ & $P^{l}$ \\
\hline \multicolumn{5}{|c|}{ Curvature Seed } \\
\hline $1 \times 10^{6}$ & 0 & 0 & $5.6 \times 10^{-7}$ & $4.5 \times 10^{-11}$ \\
\hline $2 \times 10^{6}$ & $7.5 \times 10^{-6}$ & $2.3 \times 10^{-11}$ & 1.7 & $1.2 \times 10^{-4}$ \\
\hline $5 \times 10^{6}$ & 18 & $1.5 \times 10^{-3}$ & 160 & .020 \\
\hline $1 \times 10^{7}$ & 360 & .053 & 660 & .19 \\
\hline \multicolumn{5}{|c|}{ Energy-Boosted Curvature Seed } \\
\hline $1 \times 10^{6}$ & $4.8 \times 10^{-4}$ & $5.7 \times 10^{-9}$ & 15 & $2.5 \times 10^{-3}$ \\
\hline $2 \times 10^{6}$ & 29 & $4.5 \times 10^{-3}$ & 500 & .12 \\
\hline $5 \times 10^{6}$ & 2200 & .72 & 3400 & $2.3^{\mathrm{a}}$ \\
\hline \multicolumn{5}{|c|}{ Magnetic ICS Seed } \\
\hline $1 \times 10^{3}$ & $\ldots{ }^{b}$ & $\cdots$ & 2.0 & .55 \\
\hline $2 \times 10^{3}$ & $\ldots$ & $\ldots$ & 3.5 & .73 \\
\hline $5 \times 10^{3}$ & $\ldots$ & $\cdots$ & 5.8 & .87 \\
\hline $1 \times 10^{4}$ & 0. & 0. & 7.8 & .93 \\
\hline $2 \times 10^{4}$ & 1.3 & $8.4 \times 10^{-3}$ & 10 & .96 \\
\hline $5 \times 10^{4}$ & 9.9 & .092 & 14 & .96 \\
\hline $1 \times 10^{5}$ & 22 & .18 & 17 & .96 \\
\hline $2 \times 10^{5}$ & 40 & .25 & $\ldots{ }^{c}$ & $\ldots$ \\
\hline $5 \times 10^{5}$ & 80 & .30 & $\ldots$ & $\ldots$ \\
\hline
\end{tabular}

${ }^{a}$ Replenishment is assumed in this case, to keep consistency with the normalization used for the other curvature runs; see discussion preceding equation (13) in the text.

${ }^{b}$ No cascade at these energies.

${ }^{\mathrm{c}}$ Simulations not performed; lepton DF fit poor at high energies. 
Table 4. COMPOSITE CASCADE EFFICIENCIES: PHOTONS

\begin{tabular}{|c|c|c|c|c|}
\hline \multirow[b]{2}{*}{$\gamma_{\text {beam }}$} & \multicolumn{2}{|c|}{$B=10^{12} \mathrm{G}$} & \multicolumn{2}{|c|}{$B=10^{13} \mathrm{G}$} \\
\hline & $M^{p}$ & $P^{p}$ & $M^{p}$ & $P^{p}$ \\
\hline \multicolumn{5}{|c|}{ Curvature Seed } \\
\hline $1 \times 10^{6}$ & 26 & $1.5 \times 10^{-4}$ & 26 & $1.5 \times 10^{-4}$ \\
\hline $2 \times 10^{6}$ & 1200 & $1.5 \times 10^{-3}$ & 1200 & $1.3 \times 10^{-3}$ \\
\hline $5 \times 10^{6}$ & $1.3 \times 10^{4}$ & .020 & 2800 & $3.1 \times 10^{-3}$ \\
\hline $1 \times 10^{7}$ & $9.5 \times 10^{5}$ & .095 & 320 & $2.3 \times 10^{-3}$ \\
\hline \multicolumn{5}{|c|}{ Energy-Boosted Curvature Seed } \\
\hline $1 \times 10^{6}$ & 6500 & .019 & 6500 & .016 \\
\hline $2 \times 10^{6}$ & $3.3 \times 10^{4}$ & .14 & 1200 & .030 \\
\hline $5 \times 10^{6}$ & $9.1 \times 10^{5}$ & $1.2^{\mathrm{a}}$ & 1500 & .022 \\
\hline \multicolumn{5}{|c|}{ Magnetic ICS Seed } \\
\hline $1 \times 10^{3}$ & $\ldots \mathrm{b}$ & $\ldots$ & .32 & .009 \\
\hline $2 \times 10^{3}$ & .. & .. & .36 & .005 \\
\hline $5 \times 10^{3}$ & $\cdots$ & $\cdots$ & .46 & .003 \\
\hline $1 \times 10^{4}$ & 32 & .97 & .53 & .002 \\
\hline $2 \times 10^{4}$ & 37 & .56 & .61 & .001 \\
\hline $5 \times 10^{4}$ & 270 & .56 & .73 & $4.0 \times 10^{-4}$ \\
\hline $1 \times 10^{5}$ & 1000 & .35 & $\ldots{ }^{c}$ & $\ldots$ \\
\hline $2 \times 10^{5}$ & 1200 & .20 & $\ldots$ & $\ldots$ \\
\hline $5 \times 10^{5}$ & 2600 & .099 & $\ldots$ & $\ldots$ \\
\hline
\end{tabular}

${ }^{a}$ Replenishment is assumed in this case, to keep consistency with the normalization used for the other curvature runs; see discussion preceding equation (13) in the text.

${ }^{b}$ No cascade at these energies.

${ }^{\mathrm{c}}$ Simulations not performed; lepton DF fit poor at high energies. 
Table 5. COMPOSITE CASCADES: LEPTON DF STATISTICS

\begin{tabular}{|c|c|c|c|c|c|c|}
\hline \multirow[b]{2}{*}{$\gamma_{\text {beam }}$} & \multicolumn{3}{|c|}{$B_{*}=10^{12} \mathrm{G}$} & \multicolumn{3}{|c|}{$B_{*}=10^{13} \mathrm{G}$} \\
\hline & $\bar{\gamma}^{\mathrm{a}}$ & $\gamma_{\mathrm{CM}}{ }^{\mathrm{b}}$ & $\zeta^{\mathrm{c}}$ & $\bar{\gamma}$ & $\gamma_{\mathrm{CM}}$ & $\zeta$ \\
\hline \multicolumn{7}{|c|}{ Curvature Seed } \\
\hline $1 \times 10^{6}$ & $\ldots^{\mathrm{d}}$ & $\ldots$ & $\ldots$ & 39 & 38 & 37 \\
\hline $2 \times 10^{6}$ & 160 & 80 & 1.1 & 55 & 52 & 9.8 \\
\hline $5 \times 10^{6}$ & 170 & 92 & 1.1 & 350 & 160 & 1.0 \\
\hline $1 \times 10^{7}$ & 700 & 330 & 1.3 & $\cdots$ & $\cdots$ & $\cdots$ \\
\hline \multicolumn{7}{|c|}{ Energy-Boosted Curvature Seed } \\
\hline $1 \times 10^{6}$ & 160 & 79 & 1.1 & 59 & 55 & 7.8 \\
\hline $2 \times 10^{6}$ & 150 & 81 & 1.2 & 170 & 130 & 1.6 \\
\hline \multirow[t]{2}{*}{$5 \times 10^{6}$} & 460 & 210 & 1.1 & 1500 & 590 & .60 \\
\hline & \multicolumn{6}{|c|}{ Magnetic ICS Seed } \\
\hline $1 \times 10^{3}$ & $\ldots$ & $\ldots$ & $\ldots$ & 140 & 120 & 4.5 \\
\hline $2 \times 10^{3}$ & $\ldots$ & $\ldots$ & $\ldots$ & 210 & 170 & 2.5 \\
\hline $5 \times 10^{3}$ & $\ldots$ & $\ldots$ & $\ldots$ & 370 & 260 & 1.3 \\
\hline $1 \times 10^{4}$ & $\ldots$ & $\ldots$ & $\ldots$ & 590 & 350 & .94 \\
\hline $2 \times 10^{4}$ & 110 & 62 & 1.3 & 920 & 470 & .72 \\
\hline $5 \times 10^{4}$ & 270 & 160 & 1.3 & 1700 & 890 & .53 \\
\hline $1 \times 10^{5}$ & 370 & 220 & 1.5 & 2700 & 890 & .44 \\
\hline $2 \times 10^{5}$ & 510 & 270 & 1.4 & $\ldots e$ & $\ldots$ & $\ldots$ \\
\hline $5 \times 10^{5}$ & 980 & 400 & 1.0 & $\ldots$ & $\ldots$ & $\ldots$ \\
\hline
\end{tabular}

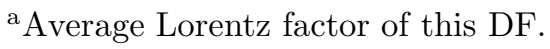

${ }^{b}$ Lorentz factor of transformation to center-of-momentum frame.

${ }^{\mathrm{c}}$ Best fit relativistic Maxwellian inverse temperature (found in $\mathrm{CM}$ frame): $\zeta=m c^{2} / k_{B} T$.

${ }^{\mathrm{d}}$ No cascade at these energies.

eSimulations not performed; lepton DF fit poor at high energies. 